\title{
A Description of the GABAergic Neurons and Axon Terminals in the Motor Nuclei of the Cat Thalamus ${ }^{1}$
}

\author{
K. KULTAS-ILINSKY,, ${ }^{*}$ C. E. RIBAK,‡ G. M. PETERSON,‡ AND W. H. OERTEL $\S$ \\ * Department of Anatomy, University of lowa, lowa City, lowa 52242, $\ddagger$ Department of Anatomy, University of California, Irvine, California \\ 92717, and §Neurology Clinic, Technical University, D-8000 Munich 80, West Germany
}

\begin{abstract}
The GABA neurons and their processes in the cat motor thalamic nuclei were identified and studied with glutamic acid decarboxylase (GAD) immunocytochemistry at both the light and electron microscopic levels. The three nuclei that comprise the motor thalamus, ventral anterior (VA), ventral medial (VM), and ventral lateral (VL), each displayed a characteristic distribution pattern of GAD-positive structures that was consistent with their afferent and intrinsic neuronal organization.
\end{abstract}

All three thalamic nuclei displayed a population of small, GAD-positive cells the dendrites of which contained synaptic vesicles and participated in complex synaptic arrays such as serial synapses, triads, and glomeruli. Based on their ultrastructural features, these GAD-containing cells were identified as local circuit neurons. In contrast, the larger, GADnegative cells were presumed to be the thalamocortical projection neurons. The axons of GAD-positive local circuit neurons could not be identified in these preparations. The number of GAD-positive dendrites in the neuropil was different for the three thalamic nuclei. In the VA and VM, the GADpositive dendrites were numerous and formed symmetric synapses with dendrites of GAD-negative cells, mainly in association with corticothalamic boutons. Within $V L$, the GAD-containing dendrites were more numerous than in VA and VM and formed synapses at influential locations on presumed thalamocortical projection neurons, such as bases of primary dendrites, and bifurcation sites of primary and secondary dendrites.

The VA and anterolateral VM nuclei that receive inhibitory GABAergic afferents from the entopeduncular nucleus and substantia nigra contained the highest concentration of large GAD-positive axon terminals. These boutons contained pleomorphic vesicles and numerous mitochondria and formed symmetric synapses and multiple puncta adherentes with dendrites and somata of presumed thalamocortical projection neurons. The size, ultrastructural features, and distribu-

Received August 9, 1984; Revised November 26, 1984; Accepted November 28, 1984

\footnotetext{
${ }^{1}$ This research was supported by grants from the Klingenstein Foundation (C. E. R.) and by National Institute of Neurolical and Communicative Disorders and Stroke Grants NS 17388 (K. K. I) and NS 15669 (C. E. R.). We gratefully acknowledge Dr. Edward G. Jones for his helpful criticism of the manuscript, $M$. Brundage, $Y$. Jhurani, and W. Wang for technical assistance, P. Reimann and B. Coons for help with illustrations, and N. Sepion and V. Yoder for secretarial assistance.

${ }^{2}$ To whom correspondence should be addressed.
}

tion of these GAD-positive boutons were similar to those features described for basal ganglia terminals in the motor thalamus of the cat. In addition, similar large-size GADpositive boutons were observed in the medial VM, which receives basal ganglia afferents exclusively from the substantia nigra. The concentration of these terminals in medial VM along the dendrites of thalamocortical projection neurons was much less than that in VA and anterolateral VM. The VL nucleus which lacks basal ganglia input did not contain any large GAD-positive boutons. Instead, small and medium size GAD-positive boutons were found in VL, where they formed symmetric synapses with dendrites of GAD-negative neurons, often in association with synaptic glomeruli.

Our findings indicate that most of the GABAergic inhibition in VA and VM is provided by basal ganglia afferents that synapse with thalamocortical projection neurons and thereby presumably control the output activity of these cells. In contrast, the VL lacks this massive extrinsic GABAergic input but has numerous presynaptic dendrites of GABAergic local circuit neurons that appear to provide a similar inhibitory control of thalamocortical projection neurons. Finally, the dendrites of thalamocortical neurons in all three nuclei receive cortical and cerebellar inputs that are often associated with GABAergic dendrodendritic synapses that probably act to modify these two sources of excitation.

The motor thalamus is traditionally recognized on the basis of its afferent and efferent connections with physiologically identified motor and premotor cortical fields. In nonprimates this thalamic region includes ventral lateral (VL), ventral anterior (VA), and ventral medial (VM) nuclei. In addition to corticothalamic projections, cerebellar and basal ganglia afferents have been ultrastructurally identified and characterized in this area of the thalamus (Grofová and Rinvik, 1974; Rinvik and Grofová, 1974b; Kultas-llinsky et al., 1978, 1980a, b, 1983). Moreover, these afferent systems terminate within the motor thalamic nuclei in a distinct topographical manner (Hendry et al., 1979; llinsky et al., 1982; llinsky and Kultas-llinsky, 1984). Another important source of afferents to the motor thalamus is the thalamic reticular nucleus (Jones, 1975; Jones, personal communication). However, the topography and ultrastructural identification of this latter projection is unknown. Since the major afferent systems to the motor thalamus have been identified, these morphological data can provide sufficient basis for the localization of neurotransmitter systems. Of particular interest are the inhibitory circuits in which GABA may be a neurotransmitter. Since relatively little is known about the transmitter chemistry as it relates to the synaptic organization of the motor nuclei of the thalamus, we have begun this analysis because such knowledge is critical for understanding the regulation of motor tunction. 

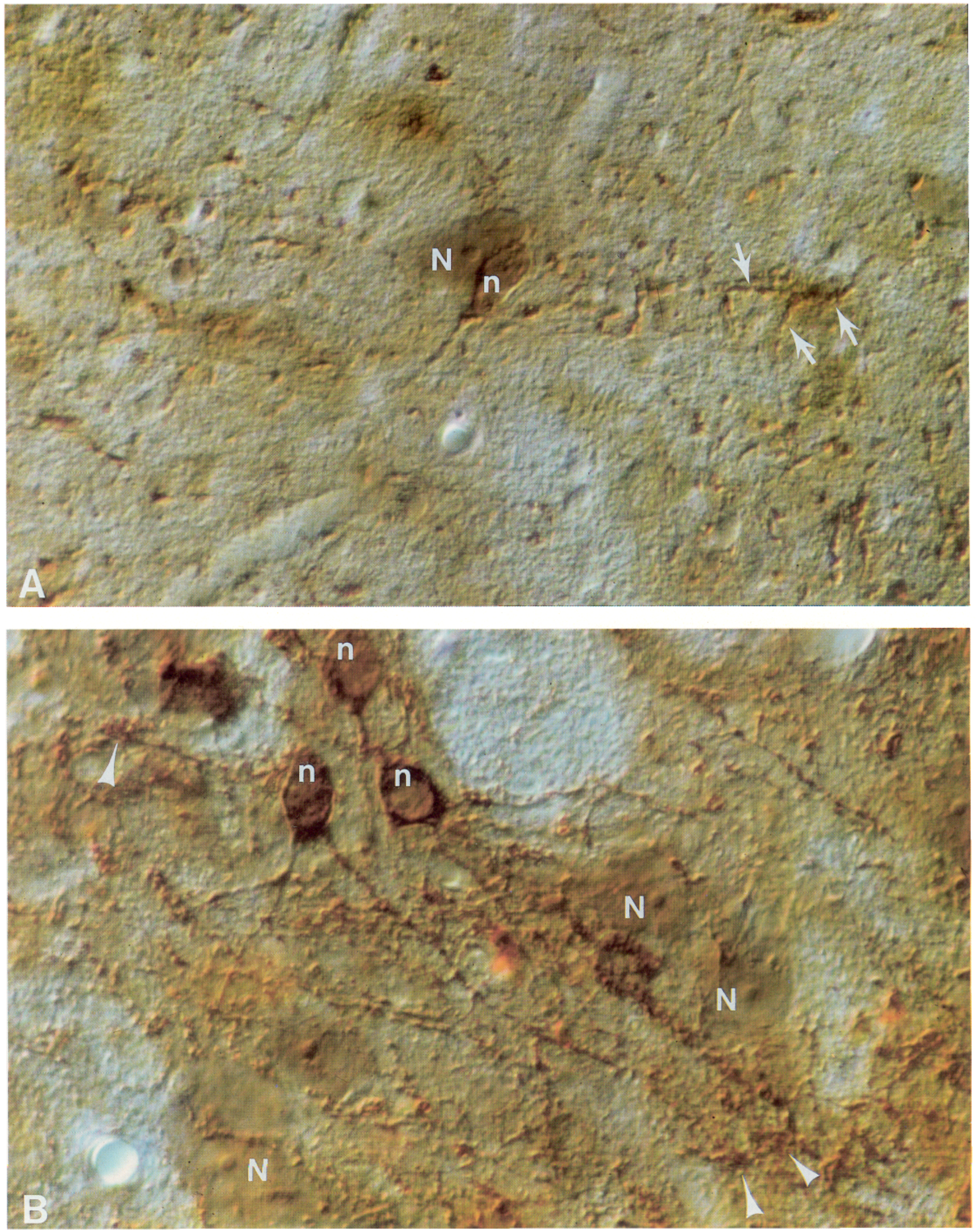

Figure 1. Nomarski photomicrographs of GAD-positive structures in the VA and VL in 40- $\mu \mathrm{m}$ Vibratome sections. A, A small GAD-positive neuron ( $n$ ) in the VA adjacent to a larger GAD-negative cell $(N)$. Arrows point to varicose GAD-immunoreactive fibers. $B, A$ group of small multipolar GAD-positive neurons $(n)$ in the VL. Slender primary dendrites of these cells give rise to much thinner secondary dendrites with irregular outlines that appear to enter clusters of GAD-positive puncta (arrowheads). N, somata of larger GAD-negative cells. Magnification $\times 2000$. 

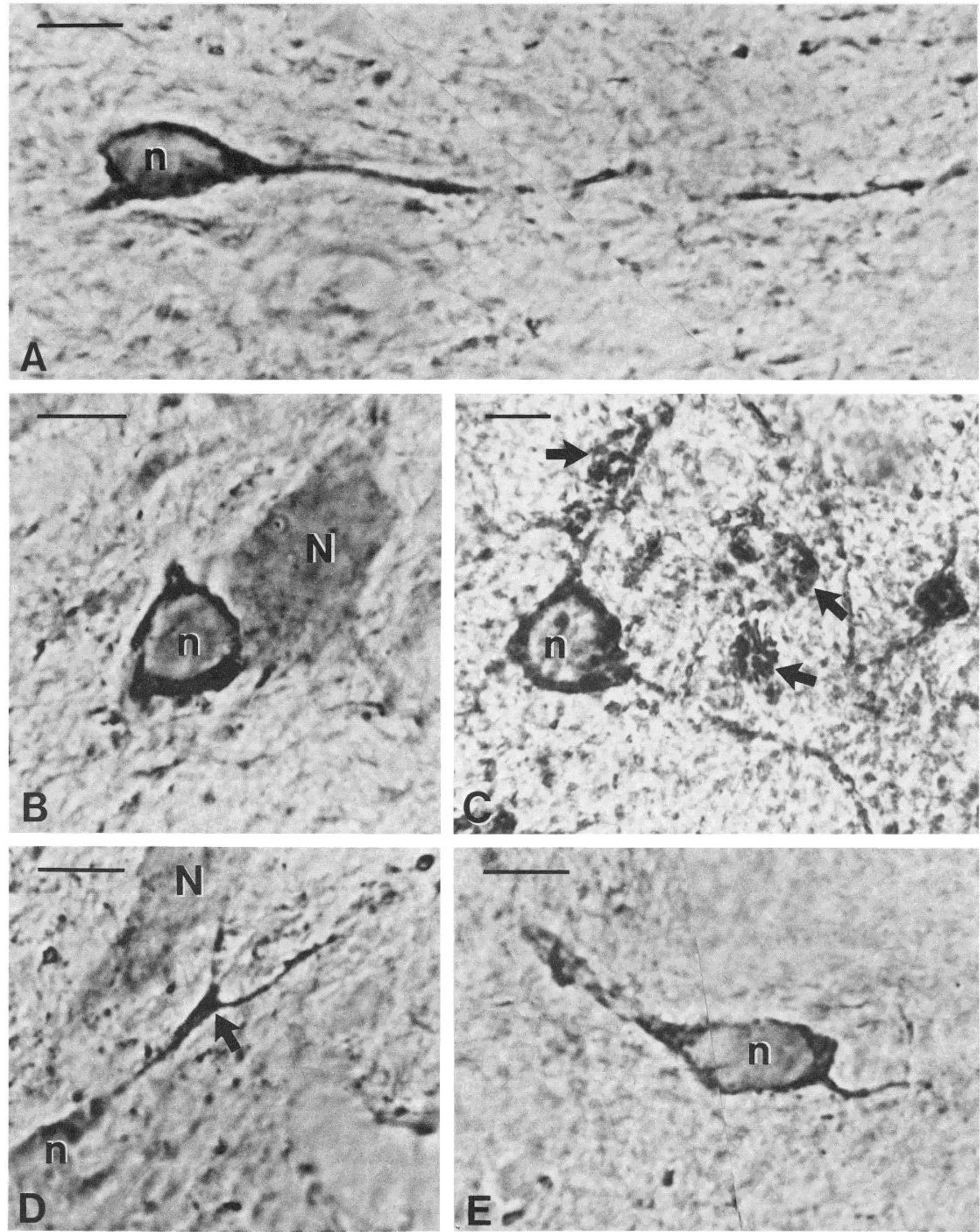

Figure 2. Phase-contrast photomicrographs taken with 100x oil objective of GAD-positive neurons and their processes in 40- $\mu \mathrm{m}$ vibratome sections. $A$ and $B$ GAD-positive small cells $(n)$ in the VA. C, A GAD-positive cell in the VL. Arrows point to clusters of GAD-positive puncta. $D$ and $E$, GAD-positive cells ( $n$ ) in the VM. The arrow points to the bifurcation of a primary dendrite. Notice the irregular outlines of the secondary dendritic branch. $N$, somata of GAD-negative neurons that appear dark due to section thickness and application of phase-contrast photomicroscopy. Bars $=10 \mu \mathrm{m}$. 

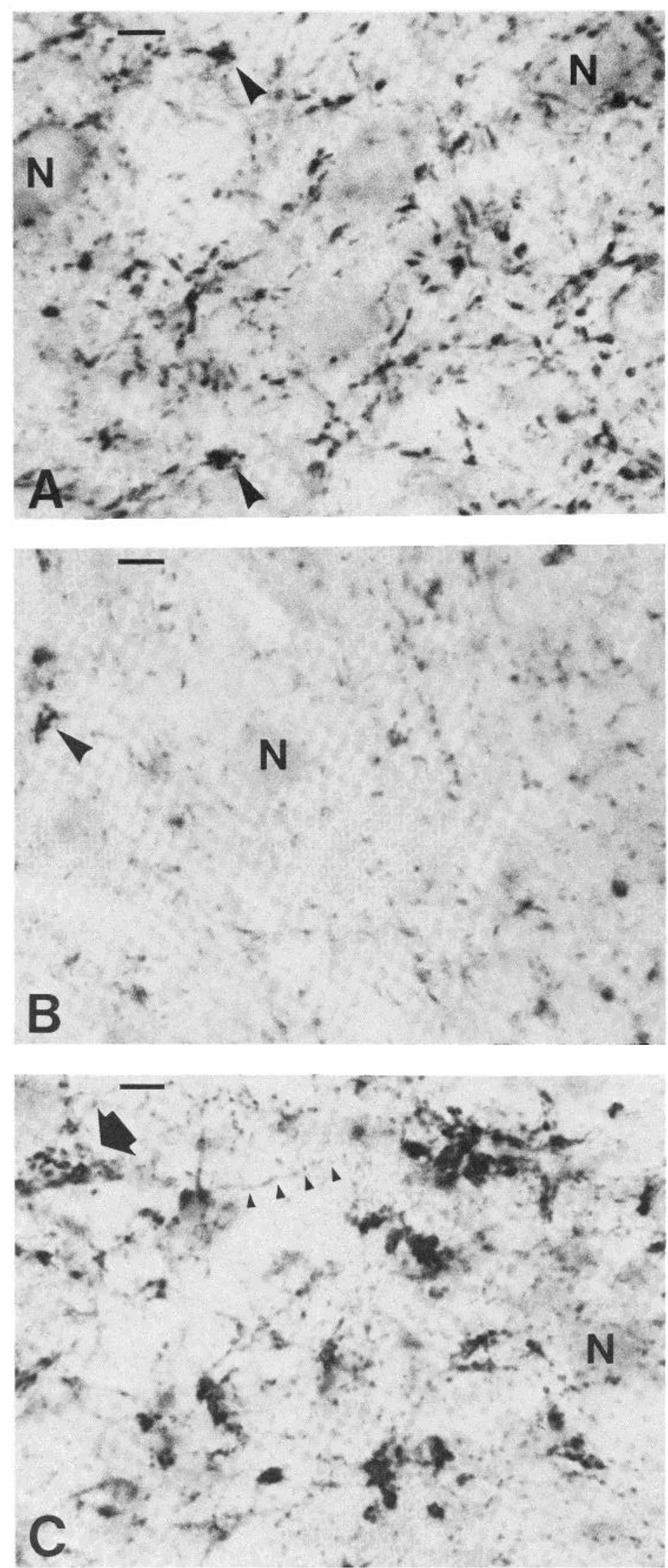

Figure 3. Photomicrographs of 40- $\mu \mathrm{m}$ Vibratome sections demonstrating differences in the density and distribution pattern of GAD-positive puncta in the motor nuclei of the thalamus. $A$ shows that the density of puncta in VA is relatively high. The puncta are usually arranged in rows, and some of them are concentrated around the somata of GAD-negative neurons $(N)$, while others form small clusters (arrowheads). $B$ shows that the density of puncta in VM is lower than that in VA. Nevertheless, puncta surround the somata of GAD-negative neurons $(N)$ and are found in clusters (arrowheads). C shows the GAD-positive puncta distribution in $\mathrm{VL}$, where they are arranged in coarse clusters. The solid arrow points to a triangular formation of a large group of puncta that are commonly found at branching sites of large GAD-negative dendrites. In the background, note the presence of numerous small puncta which often form fine varicose processes (small arrowheads). These puncta are common to all three nuclei, but they are less numerous in VA and medial VM. Bars $=10 \mu \mathrm{m}$. 

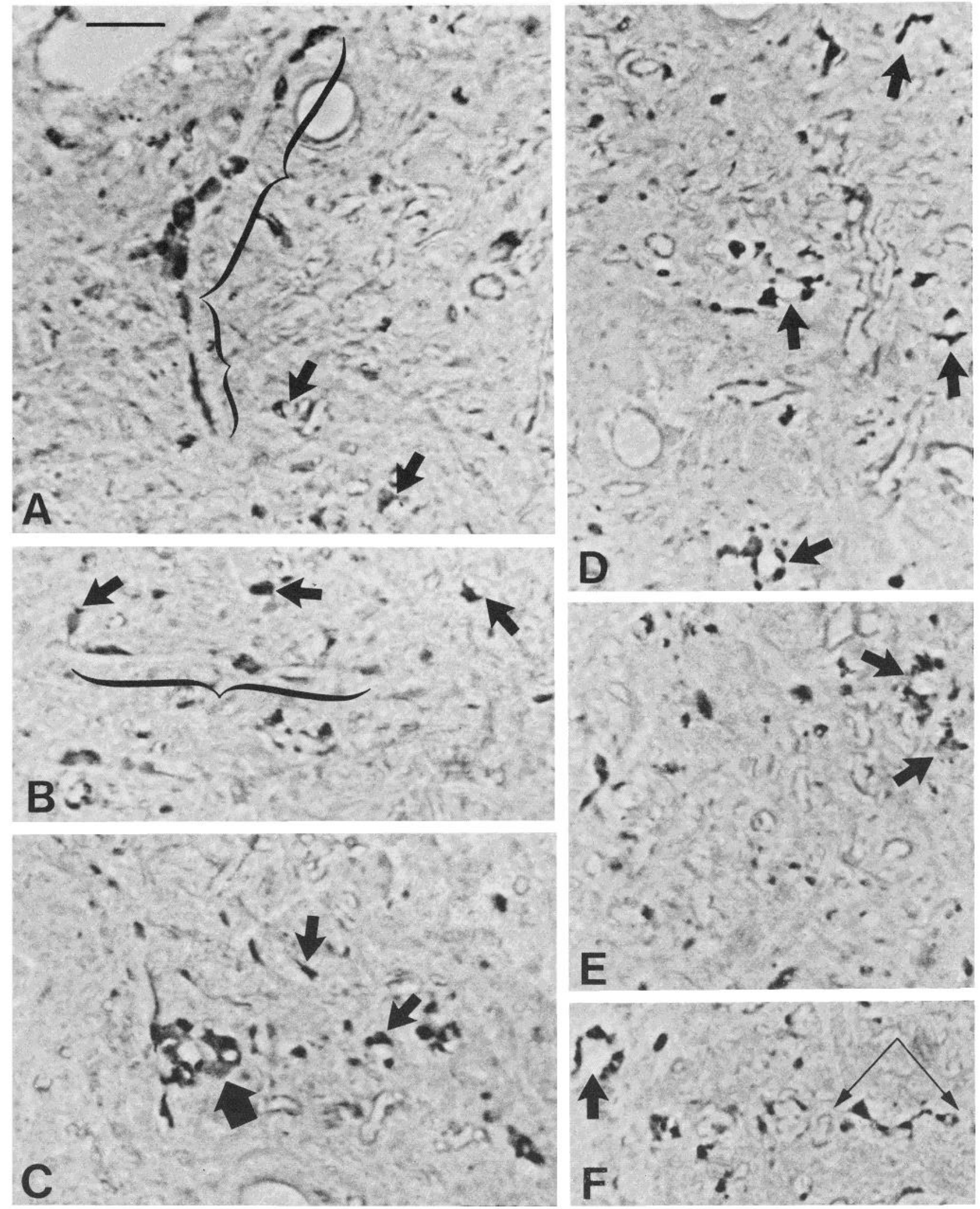

Figure 4. Phase-contrast photomicrographs of semithin Epon sections that show the differences in shape, size, and distribution pattern of GAD-positive puncta in VA, medial VM, and VL. A, Longitudinally-sectioned, GAD-negative dendrite in the VA (brackets) is apposed by large, GAD-positive puncta that almost form a continuous row. Cross-sections of GAD-negative dendrites (arrows) are apposed by individual, triangular-shaped puncta. B, Longitudinally sectioned, GAD-negative dendrite (bracket) in the medial VM is apposed by fewer GAD-positive puncta than that in VA. Large puncta (arrows) are also present in the neuropil. C, Group of GAD-positive structures in VM that appear to be a synaptic glomerulus (large arrow). It consists of several profiles of GAD-negative dendrites, GAD-positive puncta, and two fine dendritic-like, GAD-positive processes. In the adjacent neuropil, individual oval or triangular puncta (small arrows) are apposed to cross-sectioned profiles of GAD-negative dendrites similar to those in the VA. $D$ to $F, G A D$-positive puncta in the VL have irregular shapes and varying sizes. These puncta form different size groups around the profiles of GAD-negative dendrites (arrows). The adjoined arrows delineate a fine varicose GAD-positive process. Bar $=10 \mu \mathrm{m}$ for all micrographs. 

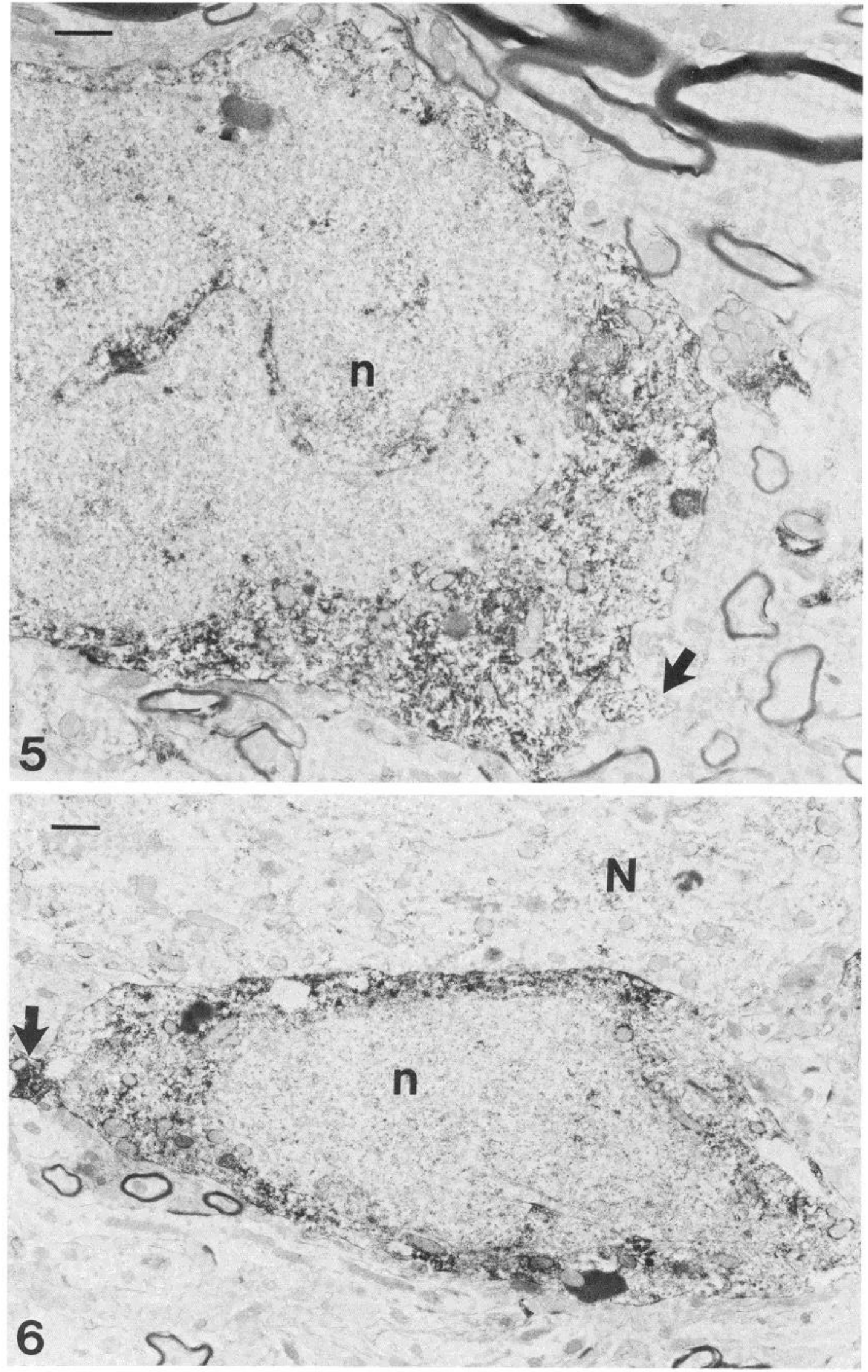

Figures 5 and 6. Electron micrographs of somata of GAD-positive neurons $(n)$ in the VA (5) and VM (6). These cells are characterized by deep indentations of the nuclear envelope (5) and are often found in close apposition to the plasma membrane of GAD-negative cells $(N)(6)$. The nuclear-to-cytoplasmic ratio in these cells is relatively high. Occasionally, GAD-positive profiles that appeared as dendrites (arrows) occurred adjacent to GAD-positive somata without synapsing on them. Bars $=1 \mu \mathrm{m}$. 

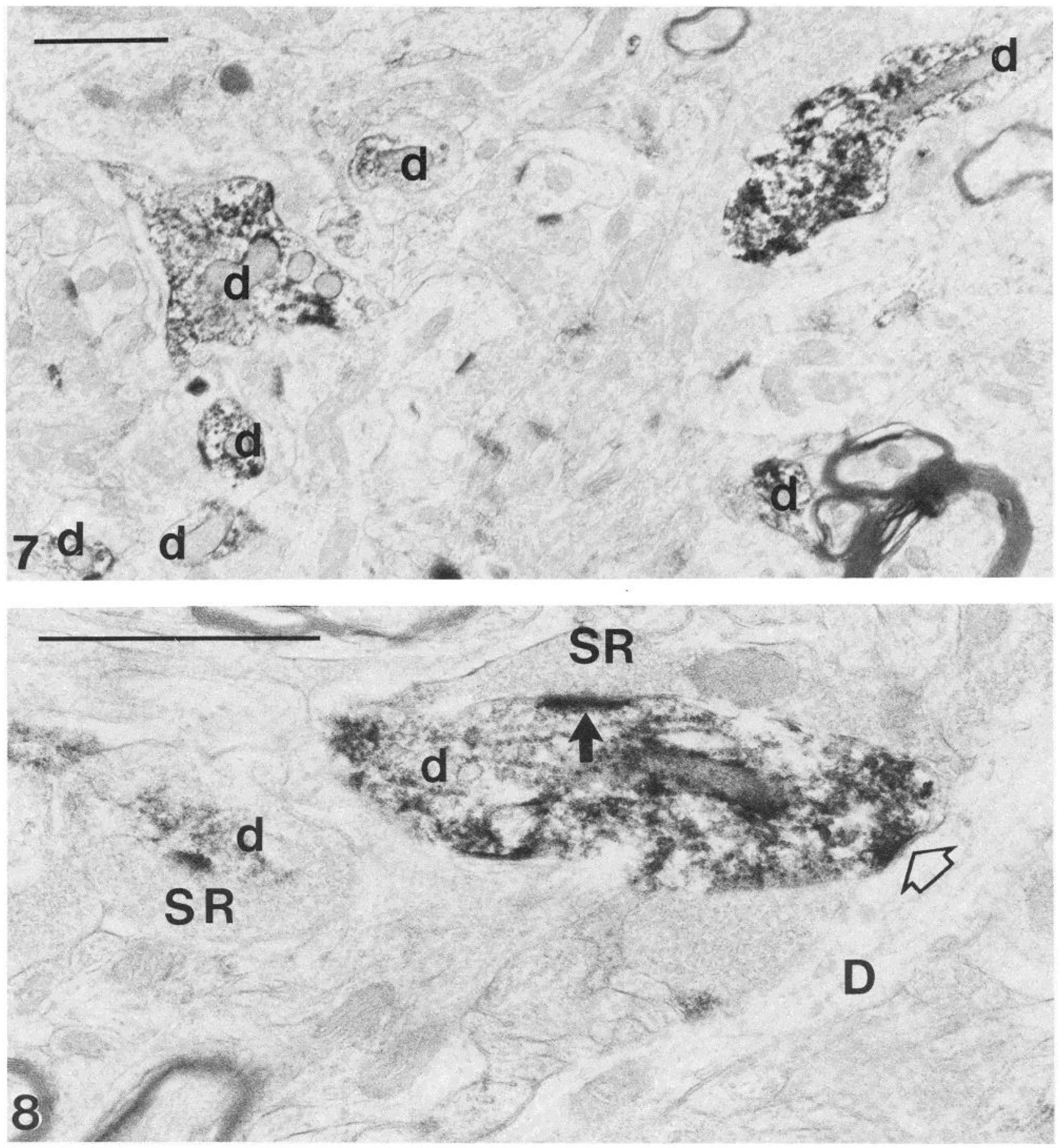

Figures 7 and 8. Examples of GAD-positive dendrites in the VA and their synaptic relationships. Bars $=1 \mu \mathrm{m}$.

Figure 7. Low-power electron micrograph demonstrates the different shapes and sizes of GAD-positive dendrites (d) in the neuropil of the VA. All of these GAD-positive dendrites contain synaptic vesicles.

Figure 8. An example of a serial synapse in the VA where a small bouton with round synaptic vesicles (SR) forms an asymmetric synapse (solid arrow) with a GAD-containing dendrite $(d)$ that makes a symmetric synapse (open arrow) with a small-diameter, GAD-negative dendrite (D). Another SR bouton contacts a small GAD-containing dendrite at the left of this micrograph. Note: the solid and open arrows have been placed on the postsynaptic side of indicated synapses in this and the following figures.

Figures 9 to 11. Examples of GAD-positive, vesicle-containing dendrites in the medial VM and their synaptic relationships. Bars $=1 \mu \mathrm{m}$.

Figure 9. A vesicle-containing GAD-positive dendrite (marked by three asterisks) has irregular outlines and accumulations of synaptic vesicles in two varicosities.

Figure 10. Three profiles of GAD-positive dendrites $(d)$ are shown. A GAD-negative, large axon terminal that contains predominantly round vesicles $(L R)$ forms asymmetric synapses (arrows) with one of these GAD-containing dendrites.

Figure 11. A GAD-positive dendrite $(d)$ involved in a serial synapse with an SR-type bouton (lower solid arrow) and a tertiary dendrite $\left(D_{3}\right)$ of a GADnegative neuron (open arrow). Another SR-type bouton contacts (upper solid arrow) the same GAD-negative dendrite.

Figure 12. A small GAD-containing bouton (asterisk) in the medial VM that forms a symmetric synapse (open arrow) with a soma of a GAD-negative neuron $(N)$. Bar $=1 \mu \mathrm{m}$. 

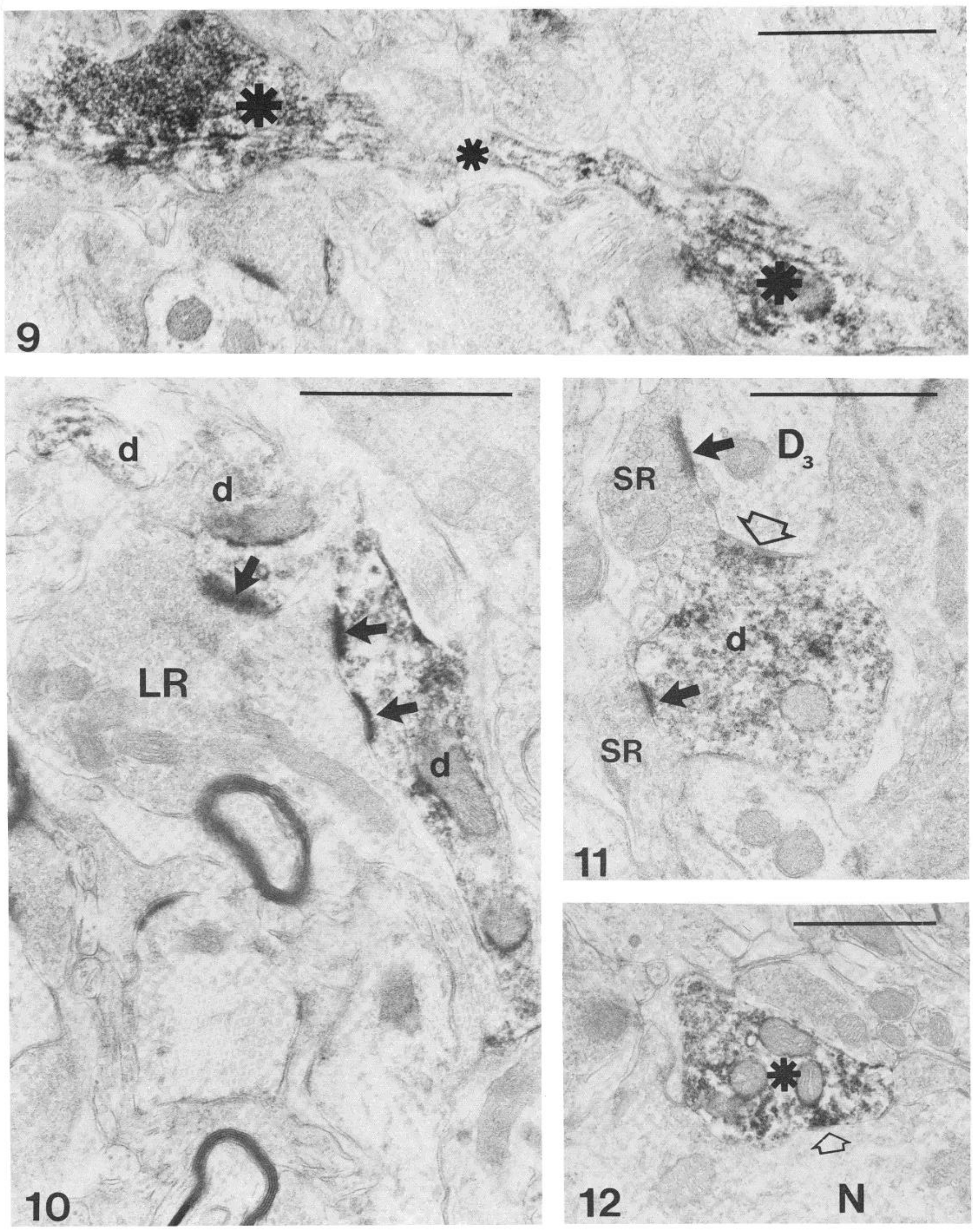

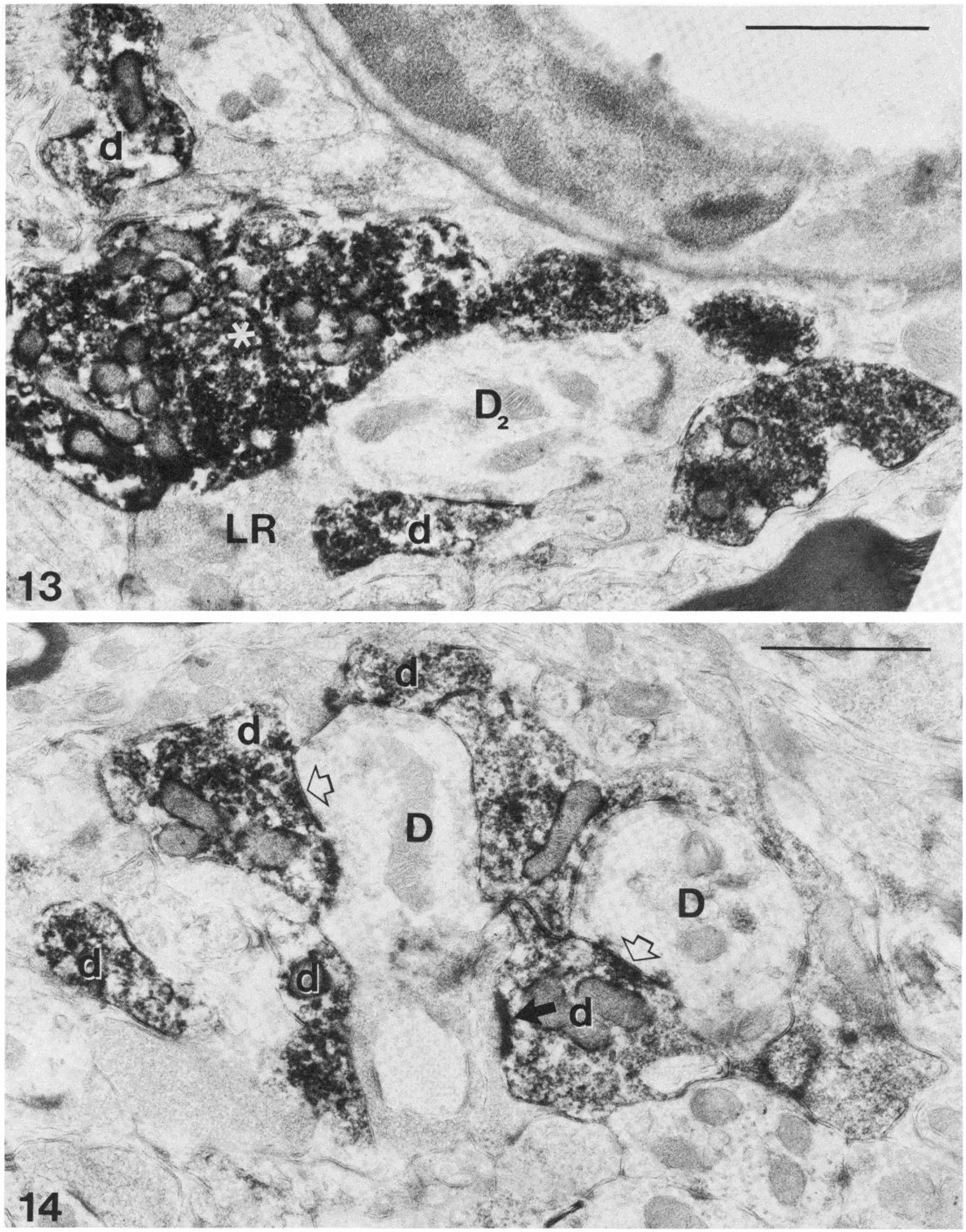

Figures 13 and 14. Examples of synaptic glomeruli in the VM. Bars $=1 \mu \mathrm{m}$.

Figure 13. A profile of a secondary dendrite $\left(D_{2}\right)$ of a GAD-negative cell apposed by an LR-type bouton, a large GAD-containing axon terminal (white 
GABA is a major inhibitory neurotransmitter in the forebrain of mammals, and the presence of moderate levels of GABA and the activity of its synthesizing enzyme, glutamate decarboxylate (GAD), has been reported in the thalamus (Fahn, 1976). Although GABA is usually thought to be localized to local circuit neurons, many brain regions (striatum, pallidum, substantia nigra, cerebellum, and thalamic reticular nucleus) display GABAergic projection neurons (Ribak et al., 1978, 1981; Houser et al., 1980). The motor nuclei of the thalamus appear to represent a unique brain region where GABAergic terminals may be derived from local circuit neurons and extrinsic sources. The evidence for the presence of GABAergic local circuit neurons is drawn from immunocytochemical studies of other specific thalamic nuclei. For example, distinct populations of small GAD-positive cells have been demonstrated in the somatosensory thalamus (Penny et al., 1983; Spreafico et al., 1983; Fitzpatrick et al., 1984) and the lateral geniculate nucleus (Ohara et al., 1983; Hendrickson et al., 1983). Since presynaptic dendrites contribute to glomeruli in all thalamic relay nuclei (Jones and Powell, 1969; Rinvik and Grofová, 1974; Harding and Powell, 1977; Kultas-llinsky et al., 1980a), and the neurons that give rise to presynaptic dendrites in some of the thalamic nuclei have been shown to contain GAD (Ohara et al., 1983; Hendrickson et al., 1983), it is likely that similar neurons in the motor thalamus are GABAergic as well.

Recent studies have suggested the possibility that GABAergic axon terminals from extrinsic sources exist in the motor thalamus. The three probable sources of this GABA input include the substantia nigra, globus pallidus, and thalamic reticular nucleus. Di Chiara et al. (1979) were first to show that GAD activity decreases significantly in the ventral thalamus following kainic acid lesions of the pars reticulata of the substantia nigra. These data were consistent with subsequent neurochemical and pharmacological findings (Kilpatrick et al., 1980; McLeod et al., 1980; Starr and Kilpatrick, 1981). In addition, physiological studies indicate that monosynaptic inhibition occurs in the VM following stimulation of the substantia nigra (Deniau et al., 1978; Ueki, 1983; McLeod et al., 1980). Furthermore, the morphological characteristics of nigrothalamic terminals, symmetric synapses and pleomorphic synaptic vesicles (Kultas-llinsky et al., 1978,1983 ) are similar to the features of identified GABAergic synapses in other brain regions (Ribak et al., 1981). Similar data exist for the pallidothalamic pathway in that: (1) the morphology of pallidothalamic synapses is similar to nigrothalamic synapses (Kultas-llinsky et al., 1983), and (2) cytochemical and electrophysiological data indicate an inhibitory, GABAergic pallidothalamic pathway (Uno et al., 1978; Penney and Young, 1981; Oertel et al., 1984). In the cat, both nigrothalamic and pallidothalamic pathways terminate mainly in VA and VM and exclude, for the most part, VL (llinsky et al., 1982; llinsky and Kultas-llinsky, 1984).

A third extrinsic source of GABAergic synapses in the motor thalamic nuclei may be the neurons of the thalamic reticular nucleus. It has been demonstrated that this nucleus sends projections to virtually all major thalamic nuclei (Scheibel and Scheibel, 1966; Jones, 1975). Electrophysiological data suggest that the projection is inhibitory and is probably driven by excitatory impulses that arrive via the axon collaterals of thalamocortical and corticothalamic libers (Schlag and Waszak, 1970; Frigyesi and Schwartz, 1972; Purpura, 1972). The terminals from the thalamic reticular nucleus have not been identified in VA, VL, or VM, but in other thalamic nuclei, they have been shown to possess the typical features of inhibitory, GABAergic terminals (Ohara et al., 1981; Montero and Scott, 1981). More direct evidence that these projections are GABAergic is gleaned from GAD immunocytochemical studies which demonstrated that most neurons in the thalamic reticular nucleus contained
GAD (Houser et al., 1980; Hendrickson et al., 1983; Oertel et al., 1983).

The present study was undertaken to identify and characterize the GAD-positive neurons and axon terminals in the VA, VM, and $V L$. Based on the data presented above, morphological differences in the GAD distribution were expected because these motor thalamic nuclei possess differences in their afferents and general synaptic organization.

\section{Materials and Methods}

Three normal adult cats were used for this study. The cats were deeply anesthesized with ketamine ( $30 \mathrm{mg} / \mathrm{kg}$, i.m.), intubated, and connected to a respirator. Then, they were perfused transcardially with $200 \mathrm{ml}$ of $0.12 \mathrm{M}$ phosphate buffer followed by 2 liters of a mixture of $4 \%$ paraformaldehyde and $0.1 \%$ glutaraldehyde in the same phosphate buffer. Immediately after the perfusion, the brains were removed and kept overnight in a fresh fixative in the refrigerator. Sections, in both frontal and sagittal planes, were made on a vibratome. Alternate series of $40-$ and $60-\mu \mathrm{m}$ sections were used for light and electron microscopic immunocytochemistry, respectively.

Both sets of sections were processed for the immunocytochemical localization of GAD according to a modification of the protocol described by Oertel et al. (1981b) using the avidin-biotin horseradish peroxidase (HRP) complex (Hsu et al., 1981; Vector Labs, Inc.) in a double-bridged technique (Ordronneau et al., 1981). Anti-GAD serum developed and characterized by Oertel et al. (1981a) was used in this study. Briefly, sections were initially incubated in $10 \%$ normal rabbit serum and $0.1 \mathrm{M} \mathrm{D}, \mathrm{L}$-lysine to suppress the background. Then sections were rinsed in Tris-saline buffer before being incubated for $18 \mathrm{hr}$ in either sheep anti-GAD serum (diluted 1:2000 with buffer) or pre-immune (control) serum. After several washes in the buffer, sections were incubated in biotinylated rabbit anti-sheep IgG, followed by several buffer washes and incubation with avidin-biotin HRP complex (ABC). After reincubation in both the biotinylated rabbit anti-sheep $\lg G$ and $A B C$, the sections were incubated in a solution containing $0.6 \%$ diaminobenzidine and $0.01 \%$ hydrogen peroxide in Tris-saline buffer. In some instances, sections were preincubated with $0.3 \%$ Triton $X-100$ in $0.05 \mathrm{M}$ Tris-saline buffer to enhance the penetration of antibodies into the sections.

The sections for light microscopy were washed again in Tris-saline buffer and placed in $0.01 \%$ osmium tetroxide for $30 \mathrm{~min}$ to enhance the intensity of HRP reaction product. After another rinse, the sections were mounted on glass slides, dehydrated, and coverslipped. Sections from the series for electron microscopy were examined with a low-power stereomicroscope, and different nuclear regions of the thalamus (VA, VM, and VL) were identified. Discs of tissue $1 \mathrm{~mm}$ in diameter were then punched out by a needle from areas within these nuclei using a technique described earlier (Kultas-llinsky et al., 1983). The tissue samples were then placed in separate numbered vials, postfixed in $1 \% \mathrm{OsO}_{4}$ for $2 \mathrm{hr}$, rinsed, dehydrated, and flat embedded in Epon. Semithin $(1 \mu \mathrm{m})$ sections were cut and studied under bright field and phase-contrast illumination in the light microscope. Thin sections were obtained and examined using a Philips EM 300 or Hitachi $\mathrm{H}$ 600 electron microscope. A total of 25 tissue blocks from different regions of $V A, V M$, and $V L$ were examined.

Adjacent vibratome sections which were not incubated for immunocytochemistry were mounted on glass slides and Nissl-stained. These sections were used to determine the boundaries of the thalamic nuclei analyzed in this study and to verify the exact location of the electron microscopic specimens that were processed for immunocytochemistry. In addition to this cytoarchitectural criterion, our delineation of motor thalamic nuclei (VA, VM, $V L$ ) utilized current data on the distribution of basal ganglia and cerebellar afferents (Kultas-llinsky et al., 1978; Hendry et al., 1979; llinsky et al., 1982; llinsky and Kultas-linsky, 1984). Thus, medial VM refers to that part of the VM nucleus medial to the mammillothalamic tract which receives nigral, but no pallidal, input. The VA includes the major ventral medial part of the classical VA that receives both nigral and pallidal projections. The lateral anterior part of the VM is also grouped with VA because it has similar afferents. Finally, $V L$ refers to the cerebellar terminal zone which lacks nigral and pallidal afferents. Previous electron microscopic data (Kultas-llinsky et al., 1981; Kultas-llinsky and llinsky, 1983) from these thalamic regions of

asterisk), and a GAD-containing dendrite (d). The three GAD-positive profiles on the right might be axon terminals based on the packing density of synaptic vesicles and the size of the mitochondria.

Figure 14. Numerous profiles of GAD-containing dendrites $(d)$ in a glomerulus formed around two GAD-negative dendrites $(D)$. Dendrodendritic synapses are indicated by open arrows. One of these presynaptic dendritic profiles receives an asymmetric synapse (arrow) from a GAD-negative bouton the nature of which remains obscure due to the odd section plane. 

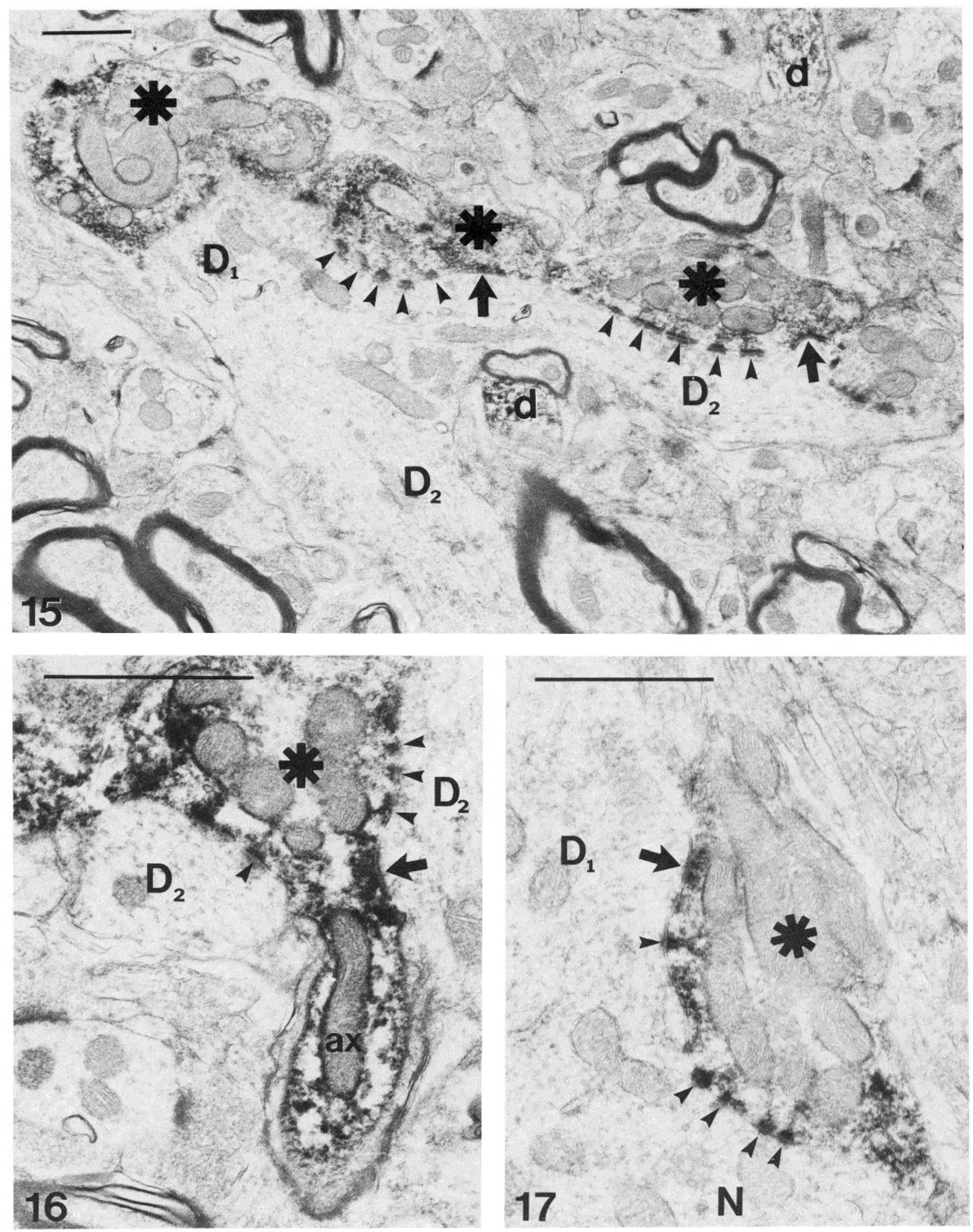

Figure 15. Low-power electron micrograph in VA shows a group of three large, GAD-positive axon terminals (asterisks) along the bifurcation site of primary $\left(D_{1}\right)$ and secondary $\left(D_{2}\right)$ dendrites. These terminals form symmetric synaptic contacts (arrows) and puncta adherentes (arrowheads). GAD-containing dendrites (d) are also evident. Bar $=1 \mu \mathrm{m}$. 
normal cats and those with HRP injections in the motor and premotor cortices were used to properly identify structures in the immunocytochemical preparations.

\section{Results}

In light microscopic preparations, the cat thalamus displays differences in the intensity of GAD immunostaining in thalamic nuclei. In general, the staining intensity is the lowest in the motor nuclei when compared to the sensory, limbic, and midline nuclear groups of the thalamus. These differences are due to variations in the number of GAD-positive cells and puncta. The term "puncta" has been often used to describe the GAD-containing axon terminals at the light microscopic level, but puncta may also represent cross-sectioned GAD-positive dendrites (Ribak et al., 1981). In most brain regions, these puncta are usually 1 to $2 \mu \mathrm{m}$ in diameter. However, the GADpositive puncta in the motor nuclei of the thalamus displayed a variety of sizes up to $5 \mu \mathrm{m}$.

Differences in GAD staining also exist between the three motor nuclei of interest. For example, VL contained more GAD-positive somata with dendrites and fewer large-size puncta than VA and VM (Figs, 1 and 3). In contrast, only subtle differences existed between VA and medial VM. In the following description, the VA and VM will first be considered together, and the VL will be described separately afterwards.

\section{Light microscopy of GAD-positive structures in the VA and VM}

GAD-positive somata were infrequently encountered in the VA and VM. These somata were round, ovoid, or fusiform (Fig. 1A, and Fig. 2, $A, B, D$, and $E$ ). The shortest diameters of cells that had nucleoli in the section plane ranged from 8 to $14 \mu \mathrm{m}$. Very often, somata of GAD-positive neurons were located adjacent to the perikarya of much larger GAD-negative cells (Fig. $1 A$ and Fig. $2 B$ ). Occasionally, a GAD-positive dendrite from one of these small cells was observed along the side of a GAD-negative soma.

Some small cells in semithin sections of VA and VM did not contain GAD-positive reaction product. Therefore, either a minority of small cells are not GABAergic or there was a limited diffusion of the antibody molecules, creating a number of false-negative images This latter possibility is more realistic, because the immunoreactive cells were confined exclusively to the surface of the tissue sections.

Usually 2 to 3 dendrites were seen to originate from the perikarya of the small.GAD-positive cells in 40- $\mu \mathrm{m}$ thick sections. In only a few instances could these dendrites be traced an appreciable distance ( 50 to $70 \mu \mathrm{m}$ ) from the soma up to the point of their first bifurcation. In these cases, the lightly stained dendrites appeared to be rather linear and of thin caliber (Fig. 2, $A$ and $D$ ). In other cases, the site of the first bifurcation was close to the perikaryon, and the proximal dendritic shafts were quite wide. The dendrites that were not in continuity with their somata were difficult to identify in the neuropil, because they intermingled with darker-staining GAD-positive puncta that were presumed to be axons.

The appearance and distribution pattern of GAD-positive puncta differed between the VA and medial VM. In the VA and the anterolateral part of the VM, the overall density of GAD-positive puncta was very high. Many puncta were dark-staining, ovoid-shaped, and arranged in rows suggestive of varicose fibers (Fig. 3A). Some of these puncta were situated along the perimeters of GAD-negative neuronal somata. In semithin Epon sections, the rows of puncta were aligned along profiles of longitudinally sectioned GAD-negative dendrites of larger cells (Fig. 4A). Another type of punctum, more round or triangular in shape, was associated with profiles of crosssectioned GAD-negative dendrites. A third type of punctum was very small (Fig. $3 A$ ), and it also appeared to form rows.

The overall density of GAD-positive puncta in the medial VM was much lower than that in VA due to the absence of continuous rows of large oval-shaped puncta (Fig. 3B). Nevertheless, large GADpositive puncta found in VM were also associated with both longitudinally and cross-sectioned profiles of GAD-negative dendrites (Fig. $4 B$ ). Some of the very small puncta in medial VM surrounded the somata of GAD-negative cells (Fig. $3 B$ ).

In addition, both nuclei displayed occasional clusters of GAD positive puncta (Fig. $3, A$ and $B$ ). In semithin Epon sections, these clusters appeared to be composed of several GAD-positive puncta of varying shapes and staining intensities and a few GAD-negative profiles. Occasionally, thin GAD-positive processes in the adjacent neuropil entered these clusters (Fig. 4C).

\section{Electron microscopy of GAD-positive structures in VA and VM}

\section{Cell bodies and dendrites}

GAD-positive reaction product was found in the perikaryal cytoplasm of small neurons, whereas the large neurons lacked staining. These GAD-positive cells had deeply indented nuclei that were surrounded by a thin rim of cytoplasm (Figs. 5 and 6). Distributed throughout the perikarya and proximal dendrites were scattered cisternae of granular endoplasmic reticulum and ribosomal rosettes. Cisternae and vesicles of the Golgi complex were also found in the perikarya and proximal dendritic processes. In addition to these typical organelles, the GAD-positive somata displayed large lysosomes of moderate electron density that were often lobulated. These lysosomes were larger and less dense than the ones found in the large GAD-negative cells. Axosomatic synapses were not found on the surfaces of GAD-positive somata in the VA and VM.

The processes observed in continuity with the GAD-positive somata were sometimes thin in caliber $(<1 \mu \mathrm{rr})$ and possessed features typical for dendrites, except that they also contained synaptic vesicles. These proximal dendrites had small clusters of 4 to 5 vesicles, while more distally, the number of vesicles in clusters increased dramatically. In fact, numerous GAD-positive dendrites, containing large clusters of synaptic vesicles, were found throughout the neuropil (Figs. 7 and 9). Most of the isolated dendrites in the neuropil were assumed to be the distal dendritic branches of the GAD-positive cells, and some could be traced in continuity for a length of several microns in the longitudinal plane of section. These processes were commonly 1 to $1.5 \mu \mathrm{m}$ in diameter and usually had smooth surfaces, while others had very irregular outlines with diameters varying from 0.5 to $1.5 \mu \mathrm{m}$. Although synaptic vesicles were present throughout the length of these processes, they accumulated in larger groups within the wider parts (Fig. 9). Mitochondria were also present in these processes, but thcy wcre rather sparse in number. Both types of distal dendritic processes (wider with regular outlines, and thinner with irregular outlines) were postsynaptic to small boutons with round vesicles that formed asymmetric synaptic contacts (Figs. 8 and 11). These axon terminals have been named the SR (small boutons, round vesicles) type boutons and most of them were shown to be corticothalamic axons (Grofová and Rinvik, 1974). Some thin, GAD-positive dendrites occasionally expanded into club-like endings which were postsynaptic to different types of axon terminals.

The GAD-positive dendrites formed a presynaptic part of numerous dendrodendritic synapses found in the neuropil of the VA and VM either singly or as a part of complex synaptic arrangements (described below). In most cases the postsynaptic structures of these synapses reflected the features of distal dendrites of large, GAD-negative cells. Also, GAD-positive dendrites were frequently observed in close apposition to the somal membrane of GADnegative cells. In a few instances, dendrosomatic synapses have been encountered at these sites in the VA and VM.

Figure 16. Myclinated GAD-containing axon (ax) in the VA giving rise to a terminal (asterisk) that forms a symmetric synapse (arrow) with a secondary dendrite $\left(D_{2}\right)$ of a GAD-negative neuron. Arrowheads indicate puncta adherentes. Bar $=1 \mu \mathrm{m}$.

Figure 17. A GAD-containing bouton in the VA (asterisk) forms a symmetric contact (arrow) with the base or proximal portion of a primary dendrite $\left(D_{1}\right)$ of a GAD-regative cell body $(N)$. Arrowheads indicale purcta adherentes. Bar $=1 \mu \mathrm{m}$. 

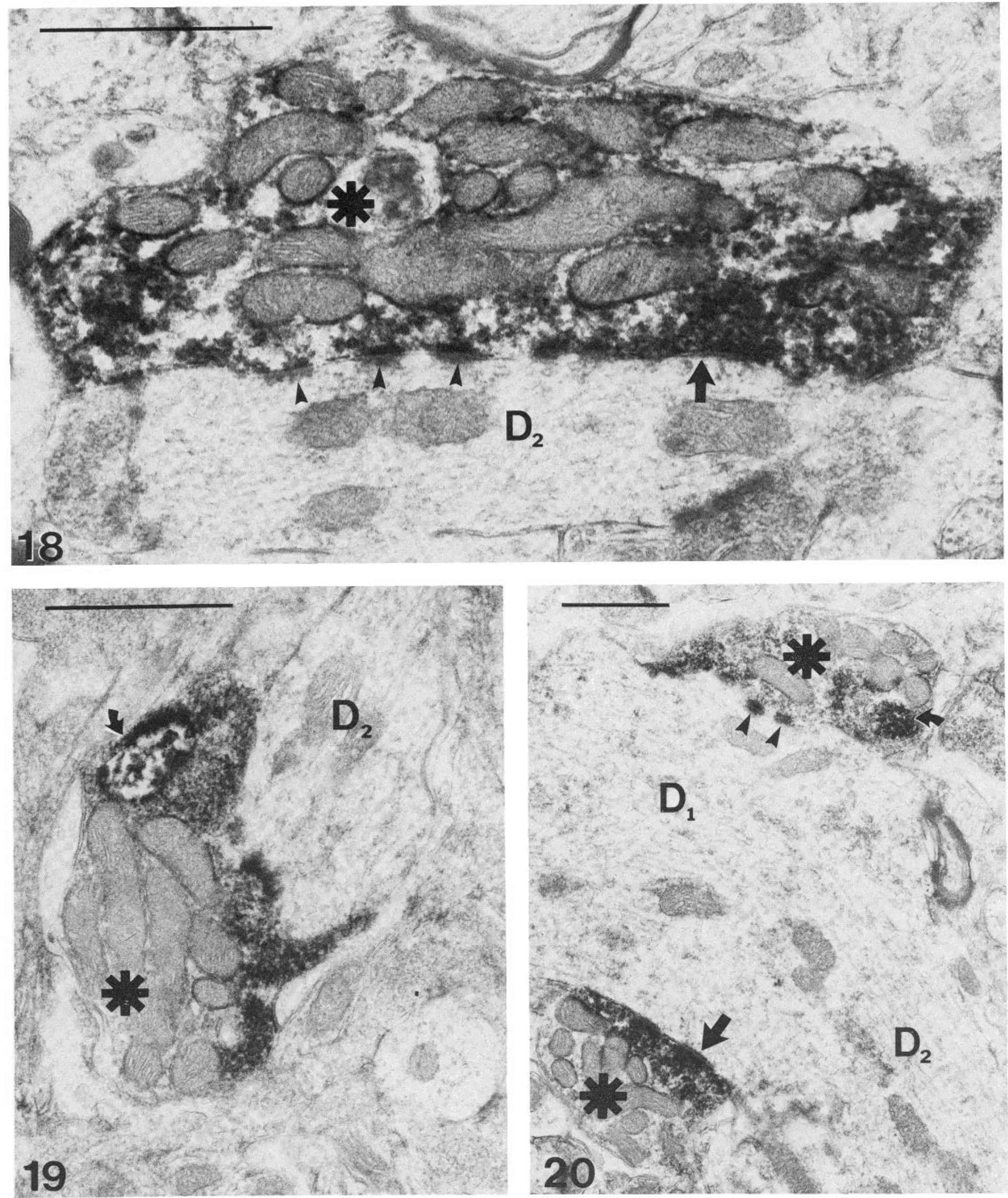

Figures 18 to 20 . Electron micrographs illustrating various synaptic sites of GAD-containing axon terminals in the VA. Small curved arrows in Figures 19 and 20 point to membrane bound GAD-containing profiles embedded within the GAD-positive boutons. All other labeling is the same as in Figures 15 to 17 . 


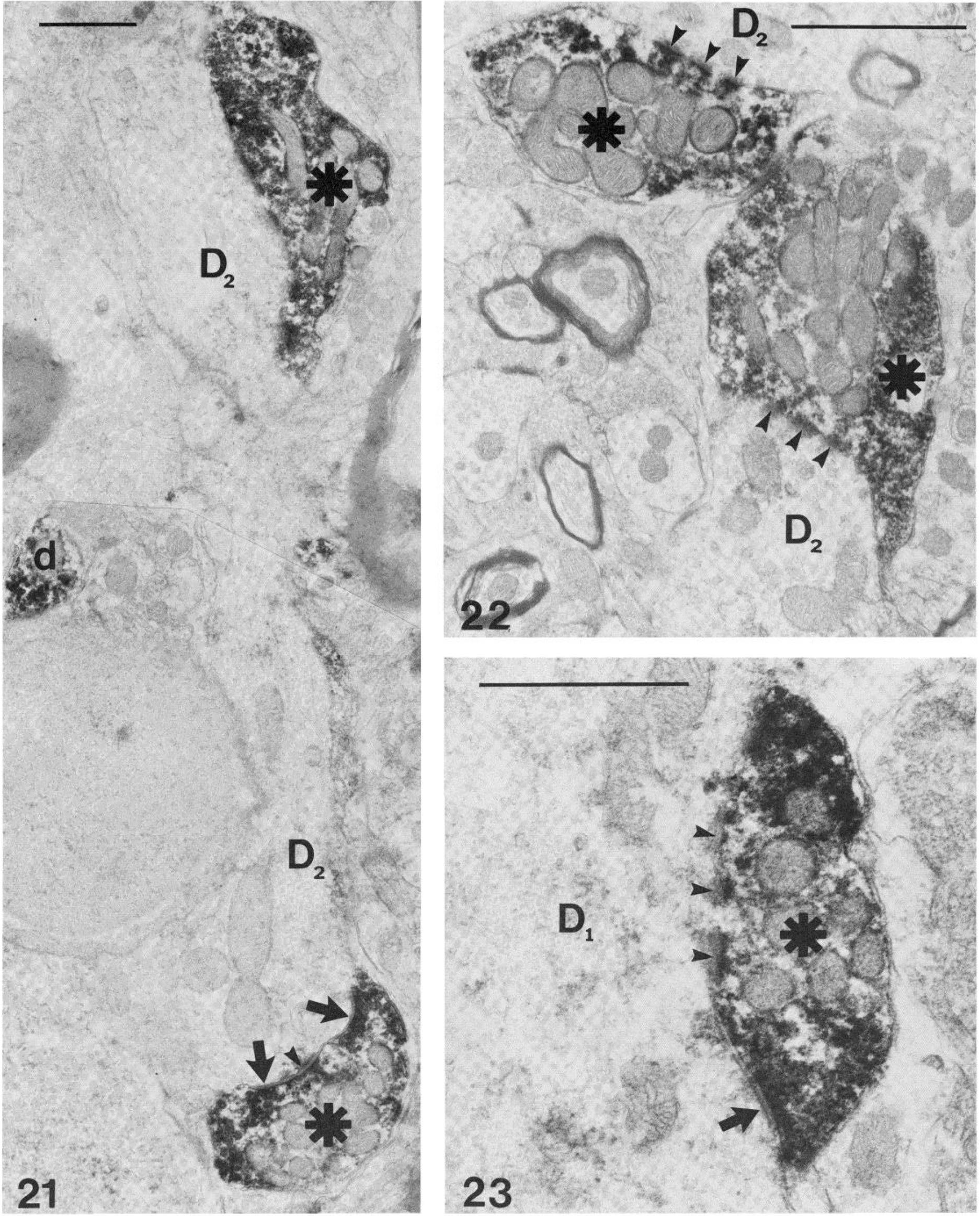

Figures 21 to 23. Electron micrographs illustrating different synaptic sites of GAD-positive boutons in the VM. The labeling of profiles is the same as in Figs. 19 to $21 . B a r=1 \mu \mathrm{m}$. 


\section{GAD-positive dendrites in complex synaptic arrangements}

Synaptic arrays with a variable degree of complexity are characteristic fealures of the neuropil in the VA and VM. The most frequently observed arrangement is the serial synapse. GAD-positive dendrites were invariably observed to participate in these complexes. In these instances, a GAD-positive dendrite was both post- and presynaptic to two different structures. Usually, an SR bouton was presynaptic to the GAD-positive dendrite, which in turn formed a symmetric synaptic contact with an adjacent unlabeled profile tentatively identified as a distal dendrite of a large GAD-negative cell (Figs. 8, 11, and $36 \mathrm{~A}$ ). In a few instances, the somata of GAD-negative neurons were postsynaptic to GAD-containing dendrites in such serial synapses.

Another type of terminal involved in synaptic relationships with GAD-positive dendrites was a large bouton with predominantly round vesicles and several mitochondria. Such boutons were identified as LR (/arge boutons, round vesicles) boutons, and many of them are the terminals of ccrebcllar afferents (Rinvik and Grofová, 1974b; Kultas-llinsky et al., 1980a, b). In the VA and VM (excluding its posterolateral region), these LR terminals were not as common as the SR type, but they also formed asymmetric synapses with GADpositive dendritic profiles (Fig. 10). Usually one of these large terminals contacted one to two profiles of GAD-positive dendrites and at least one GAD-negative dendrite. In favorable section planes, symmetric synapses were observed between these two latter types of profiles, thereby constituting a synaptic triad that is a typical relationship for cerebellar boutons in the VA and VM (see diagram in Fig. 36B).

Numerous GAD-positive profiles were found in synaptic glomeruli (Figs. 13 and 14). In the cat motor thalamus, glomeruli are complex synaptic arrangements composed of dendrites and axon terminals of various origins (Rinvik and Grofová, 1974a, b; Kultas-llinsky et al., $1978,1980 a, b, 1983$ ). Most of the GAD-containing profiles in the glomeruli were identified as enlargements of GAD-positive dendrites, and they formed symmetric contacts with GAD-negative dendrites. An LR bouton was frequently observed as a component of a glomerulus, where it was presynaptic to both GAD-positive and GAD-negative dendritic profiles. In addition, medium and large GADpositive axon terminals (described in detail below) were also found in these complexes (Fig. 13). It should be mentioned that the identification and synaptic relationships of many GAD-positive profiles in the glomeruli were not always clear, due to heavy deposits of reaction product and unfavorable section planes.

\section{GAD-positive axons and terminals}

A number of GAD-positive myelinated axons of varying diameters were observed in the neuropil of VA and VM. These axons often gave rise to GAD-positive terminals that formed symmetric synapses (Fig. 16).

The most characteristic feature of the neuropil in the VA and VM was the presence of numerous medium-to-large GAD-positive synaptic boutons. The appositional length of these boutons with postsynaptic dendrites was usually within 2 to $5 \mu \mathrm{m}$. In a few instances, very long (up to $10 \mu \mathrm{m}$ ), oval-shaped GAD-positive boutons filled with vesicles, microtubules, and mitochondria were observed in the neuropil. All of these medium-to-large GAD-positive boutons formed symmetric synaptic contacts, displayed numerous puncta adherentes with the postsynaptic structures, and usually contained large numbers of mitochondria (Figs. 13 and 15 to 23). The distribution pattern of these boutons was different for the VA and medial VM. In the former, almost continuous rows of these boutons were often observed to appose GAD-negative dendrites (Fig. 15). In contrast, fewer boutons made contact with the same dendrite in VM (Fig. 21), and therefore the overall density of the dendritic coverage by GADpositive boutons was much lower than in the VA.

The synaptic sites of the GAD-positive boutons in the two nuclei were quite similar. The majority of terminals contacted primary (Figs. $15,17,20$, and 23), secondary (Figs. 15, 16, 18, 19, 21, and 22), and, to lesser degree, tertiary dendritic branches of GAD-negative neurons. The GAD-immunoreactive boutons were often found in groups surrounding the bifurcation sites of different-order dendrites of GAD-negative cells (Figs. 15 and 20). Although some GADpositive terminals contacted GAD-negative somata, contacts with axon initial segments were not observed. In the VA, GAD-positive boutons formed synapses with the somata of GAD-negative cells, as well as the bases of primary dendrites (Fig. 17). Very often several of these boutons were situated next to one another and, in addition to forming symmetric synapses, they also displayed numerous puncta adherentes. In general, the frequency of these axosomatic synapses formed by GAD-positive terminals was low when cornpared to the axodendritic contacts. In the medial VM, GAD-positive terminals were commonly located around the bases of proximal dendritic shafts in a manner similar to that found in the VA (Fig. 23), and only rarely were they observed to form symmetric synapses with somata of GAD-negative cells. However, the boutons found to synapse with somata did not exhibit the typical features of the majority of GAD-positive terminals in the VA and VM, because they were relatively small, lacked puncta adherentes, and contained few mitochondria (Fig. 12). Since the packing density of synaptic vesicles in these presumed axosomatic profiles was lower than that in most GAD-positive boutons, it is possible that some of these profiles are actually GAD-positive dendrites.

Another interesting observation was the possible synaptic contact between GAD-positive boutons and GAD-positive dendrites. Frequently, GAD-positive boutons had protrusions of small GAD-positive profiles within them (Figs. 19, 20, and 36C). The membrane appositions were suggestive of synaptic contacts between these two GABAergic structures. However, the ultrastructural features of synaptic contacts between them were obscured due to heavy deposits of immunocytochemical reaction product on both sides of these membrane appositions.

\section{Correlation between the light and electron microscopic results in the VA and medial VM}

A carcful comparison of the shapes and sizes of the GAD-positive structures in light and electron microscopic preparations has suggested certain anatomical correlates. First, the smallest puncta observed in the light microscope are probably the distal dendritic processes of the GAD-positive neurons. The large puncta of oval, round, or triangular shapes are apparently the medium-to-large GADpositive axon terminals with numerous synaptic contacts, puncta adherents, and mitochondria. The differences in the distribution pattern of these latter puncta in the VA and VM correlate with differences in the density of these medium to large terminals in the neuropil and reflect the different concentrations of the GAD-positive boutons on the dendrites of the unlabeled, large cells. The varicose nature of the fibers associated with these large puncta is partially due to the "en passant" characteristic of many of the GAD-positive boutons in VA since, at the ultrastructural level, thin, GAD-positive axonal processes connected adjacent boutons that were aligned along the same dendrite. Finally, the counterparts of the light microscopic clusters of the GAD-positive puncta are probably the synaptic glomeruli, where many of the GAD-positive profiles are the terminal enlargements of GAD-positive dendrites and some are GAD-positive axon terminals.

\section{Light microscopy of GAD-positive structures in VL}

The somata of GAD-positive neurons in the VL were more numerous than those in the VA and medial VM. This difference was observed in both sagittal and frontal planes, where these nuclei were included in the same section. This finding indicates that the difference was not caused by variations in the immunocytochemical procedure. Secondly, the somata of GAD-positive cells in VL were slightly larger than those in VA and VM (mean shortest diameter, $13.2 \mu \mathrm{m} ; \mathrm{SD}=2.02$ ). Finally, most of the GAD-positive cells in $\mathrm{VL}$ were small, multipolar neurons with well-pronounced dendrites that could be traced for considerable distances (Fig. $1 B$ and Fig. 2C) With lew exceptions, these proximal dendrites were slender and 
had a relatively uniform width. The somata of GAD-negative neurons were about twice the size of the GAD-positive somata. However, all of the small cells were not GAD-positive. This finding is similar to that in VA and VM and is probably due to antiserum penetration problems, because small GAD-negative cells were usually found in the depth of the vibratome sections. In the semithin Epon sections, most cytological features of GAD-positive somata were similar to those of GAD-positive somata in the VA and VM, except that those in VL had a wider band of perikaryal cytoplasm.

The density of GAD-positive puncta in the VL was high as in VA, but the distribution pattern was different from that in the VA and VM (Fig. $3 C$ ). In the VL the majority of puncta were arranged in coarse clusters (Fig. $2 \mathrm{C}$ and Fig. $3 \mathrm{C}$ ). In the background, very fine puncta, similar to the smallest ones observed in the VA and VM, were quite numerous and often arranged in rows that suggested the presence of very fine varicose processes (Fig. $3 C$ and Fig. $4 F$ ). In contrast, large single puncta typical of the VA and VM were not detected in the VL. In semithin Epon sections (Fig. 4, D to $F$ ) the majority of the puncta possessed varying shapes and sizes and usually formed clusters around large profiles of GAD-negative dendrites. Frequently very fine puncta could be resolved within these clusters (Fig. 4, D and $E$ ). In general, even at the light microscopic level, the appearance of these clusters in the VL was quite different from those in the VA and VM.

\section{Electron microscopy of GAD-positive structures in the VL}

\section{Cell bodies and dendrites}

The ultrastructural features of the perikarya of GAD-positive cells in the VL were similar to those in the VA and VM. However, axosomatic synapses were different in that somata and proximal dendritic shafts of GAD-positive cells in the VL were contacted by SR boutons that formed asymmetric synapses (Fig. 24). The majority of the GAD-positive dendrites observed in the neuropil of the VL were 1 to $1.5 \mu \mathrm{m}$ in diameter. These distal dendrites had either smooth contours or were of varicose shape (Fig. 25). They were postsynaptic to both the large and small unlabeled terminals and presynaptic to GAD-negative dendrites as described for those in the VA and VM. It is interesting to note that GABAergic, dendrodendritic synapses in the VL were very often found at strategic locations, such as bifurcation sites of the dendrites of GAD-negative cells (Figs. 30 and 31). By comparison, similar sites in the VA and VM were usually occupied by GAD-positive boutons. On numerous occasions, GAD-positive dendrites were found in close apposition to somata of GAD-negative neurons (Fig. 27), but it has not been possible to demonstrate dendrosomatic synapses in the VL.

In many instances, the synaptic relationships of GAD-positive varicose processes with other elements of the neuropil were not clear. Therefore, their identification as dendrites or axons of GADpositive cells was not possible. As to their ultrastructural features, the varicose processes were not different from those of the processes identified as dendrites. Both contained numerous microtubules, variable size clusters of synaptic vesicles, and occasional mitochondria. The presence of ribosomes, which is often used as a specific feature to distinguish between axons and dendrites, could not be detected in any of these processes due to the heavy deposits of reaction product that obscured the fine ultrastructural details. Thus, the exact identification of some of the varicose GAD-containing processes in the VL remains obscure. However, it is apparent that they are the counterparts of the finest puncta that are arranged in rows in light microscopic preparations.

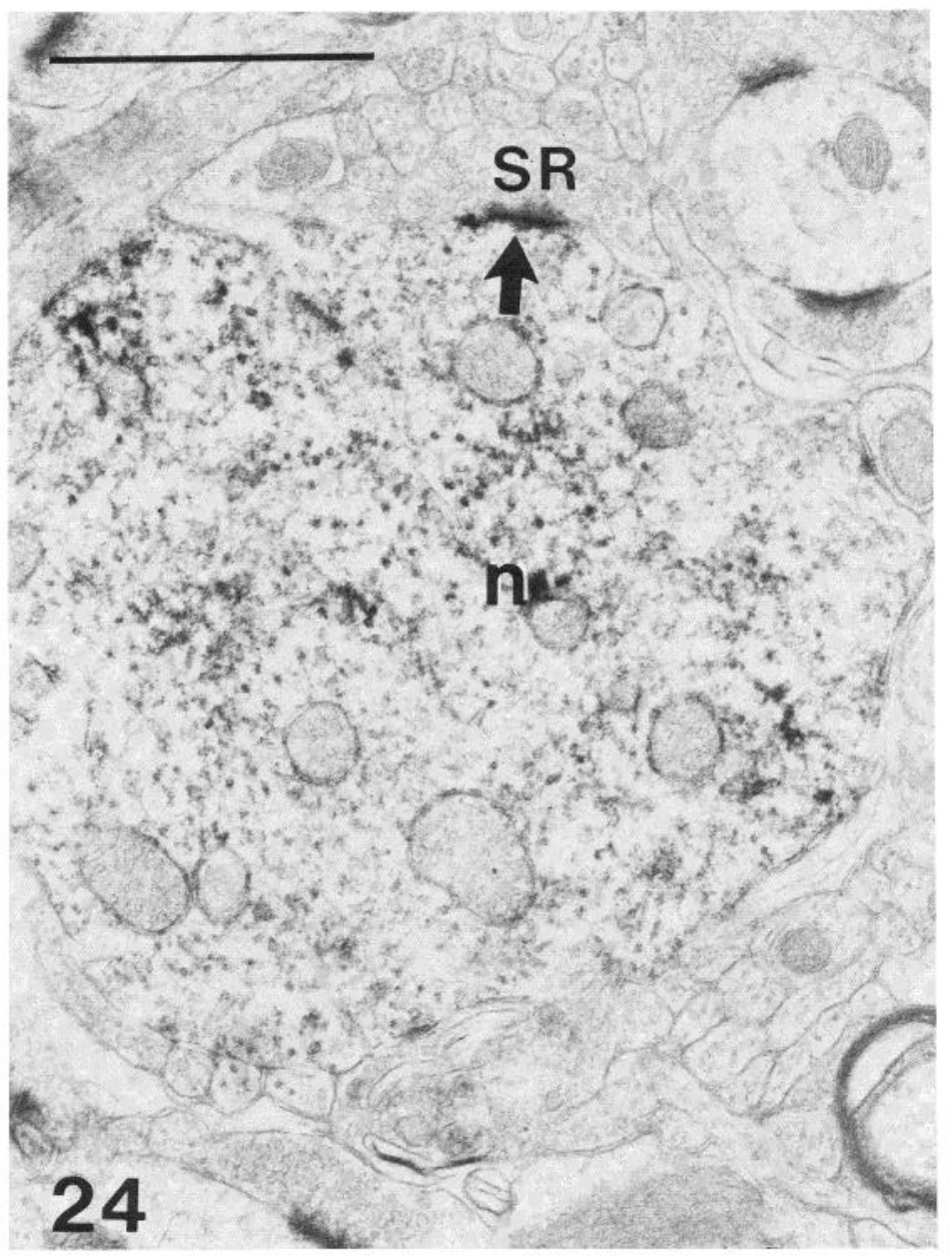

Figure 24. Distal part of a soma $(n)$ or proximal dendritic shaft of a GAD-positive cell in the VL that receives an asymmetric synapse (arrow) formed by an SR-type bouton. Bar $=1 \mu \mathrm{m}$. 

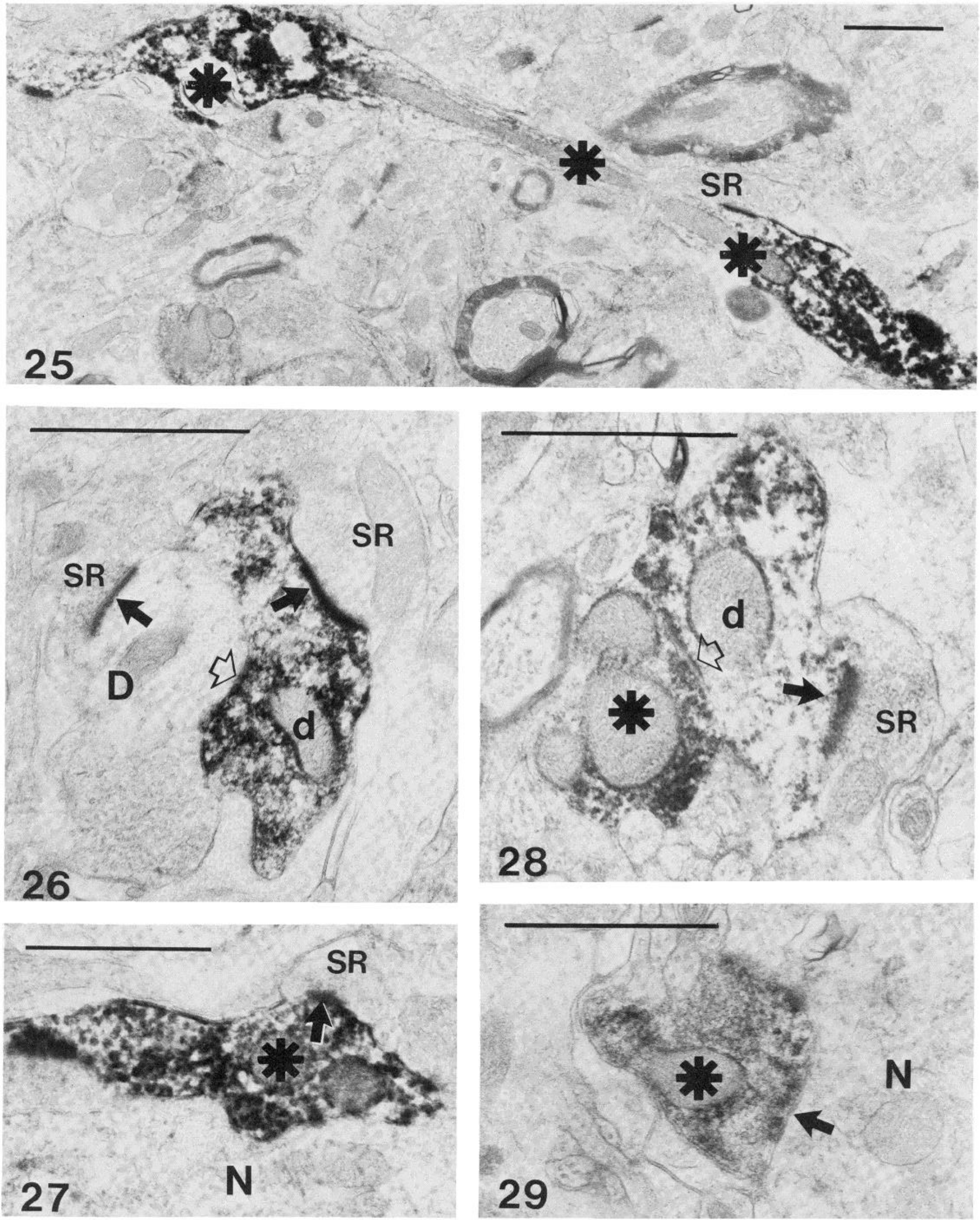

Figures 25 to 28. Examples of synaptic relationships formed by GAD-positive dendrites in the VL. Bars $=1 \mu \mathrm{m}$.

Figure 25. A varicose dendrite in the VL (asterisks) is postsynaptic to an SR-type bouton. 


\section{GAD-positive dendrites in complex synaptic arrangements}

GAD-positive dendrites in $\mathrm{VL}$ were found to be involved in serial synapses in a manner similar to that in VA and VM; they were postsynaptic to unlabeled SR boutons and presynaptic to unlabeled dendrites (Fig. 26). Large numbers of GAD-positive profiles in the $V L$ were found within the synaptic glomeruli, and most of these profiles were identified as dendrites. Each glomerulus in the VL contained 1 to 3 LR-type axon terminals that were presynaptic to both GAD-negative and GAD-positive dendrites. Within the glomeruli, 2 to 6 GAD-positive dendritic profiles of varying size could be found in association with a single LR bouton (Figs. 33 and 36D). The glomeruli were usually associated with secondary and tertiary dendrites of GAD-negative cells. Most of the GAD-positive dendrites in the glomeruli contained synaptic vesicles and formed symmetric synaptic contacts with the same GAD-negative dendrites that were postsynaptic to LR boutons. In addition, typical triads with LR boutons and GAD-positive dendrites were frequently observed outside the glomeruli (Fig. 30).

Although there are many basic similarities to thalamic glomeruli, those in the VL possessed a number of features that made them different from the glomeruli in the VA and VM. The main differences were the: (1) absence of large GAD-positive boutons; (2) presence of several LR boutons; and (3) large number of profiles of GADcontaining dendrites associated with each LR bouton, as well as a wide range of diameters (from 2 to $0.2 \mu \mathrm{m}$ ) displayed by these profiles. Finally, the glomeruli represented the most typical feature of the neuropil in the $\mathrm{VL}$, whereas they were relatively rare in VA and VM.

\section{GAD-positive axons and terminals}

A few GAD-positive myelinated axons of varying diameter were observed in the VL (Fig. 31). However, it was not possible to determine whether these axons terminated in the nucleus itself or were fibers of passage.

It should be stressed that in contrast to VA and VM only a small number of GAD-positive structures in the VL could be definitely identified as axon terminals. Moreover, the ultrastructural features and synaptic sites of the GAD-positive axon terminals in the $V L$ were different from those in the VA and VM. Based on their ultrastructural features, these boutons in the VL could be divided into two categories. One group consisted of very small boutons $(0.5$ to $1 \mu \mathrm{m}$ in length) that contained one or two mitochondria and very smalldiameter elongated synaptic vesicles (Figs. 32 and 34). These boutons formed symmetric synapses with dendrites of GAD-negative cells and were usually observed outside, but in close proximity to, the glomeruli. The concentration of immunoreaction product in these boutons was rather moderate when compared to that found in the neighboring, GAD-positive, dendritic profiles.

Another category consisted of axon terminals of rather variable morphology. These GAD-positive boutons were usually not larger than $2 \mu \mathrm{m}$ in length, contained no more than 4 mitochondria per profile, and did not display puncta adherentes. These boutons formed symmetric synapses with GAD-negative somata and dendrites of different diameter (Figs. 29 and 35)

Finally, evidence in favor of the existence of synaptic contacts between two GAD-positive structures was also found in the VL (Fig. 28). In these cases, the postsynaptic element was a dendrite, while the presynaptic element appeared to be an axon terminal that belonged to the second category of boutons as described above.

\section{Discussion}

The results of these experiments demonstrate the presence of various $\mathrm{GAD}$-containing structures in the motor thalamus of the cat. Since the localization of GAD to specific neurons indicates that they are GABAergic (Ribak et al., 1981), the data also provide new information on the organization of inhibitory GABAergic synaptic circuits in each of the three thalamic nuclei studied.

GABAergic synaptic circuits. One of the significant findings of the present study is that each nucleus of the motor thalamus displays its own characteristic pattern of GABAergic synaptic circuits. This pattern correlates well with the known synaptic organization and pattern of afferent termination. For example, the regions that displayed the largest number of medium-to-large GAD-positive terminals were the VA and anterolateral VM, where the axons of nigro- and palidothalamic pathways terminate (llinsky et al., 1982). In contrast, the medial VM, which receives only one of these basal ganglia projections (from the substantia nigra), has a smaller number of GAD-containing boutons. In both VA and VM, the size, ultrastructural features, and distribution of the GAD-containing boutons correlated well with the same features described for basal ganglia terminals in the motor thalamus of the cat (Kultas-llinsky et al., 1983). It is also interesting to note that the GABAergic synapses formed by these boutons in the VA and VM occupy the most strategic positions to influence the activity of thalamocortical projection neurons, such as somata, bases of primary dendrites, and the branching sites of different order dendrites. Therefore, these results indicate that the GABAergic inhibition in the VA and VM is predominantly of basal ganglia origin, and the strategic position of these GABAergic synapses may allow the basal ganglia terminals to dominate the activity of thalamocortical projection neurons by being more effective than terminals derived from other sources.

Consistent with this finding is the lack of large GAD-positive boutons in the $V L$ which does not receive basal ganglia projections. Instead the VL has numerous GABAergic dendrites that represent the most conspicuous feature of the neuropil, with only a moderate number of GAD-positive terminals present. Most of the dendrodendritic synapses formed between these GABAergic dendrites and the dendrites of presumed thalamocortical projection neurons are found at dendritic branching sites, a location at which basal ganglia terminals are found in VA and VM. This difference suggests that inhibitory circuits in these nuclei are organized differently.

GABAergic thalamic cell types. A consistent finding for all three thalamic nuclei was the labeling of the small cells with GAD immunocytochemistry. The available evidence suggests that these cells are local circuit neurons. Golgi studies in the cat thalamus have demonstrated two basic cell types. For example, in VA, VM, and $V L$, large type I cells and small type II cells were identified as thalamocortical projection neurons and local circuit neurons, respectively (Kiss and Tömböl, 1972; Rinvik and Grofová, 1974a). The latter authors also demonstrated that the smaller cells gave rise to vesicle-containing dendrites. In addition, two similar populations of cells were recognized in VA, VM, and VL following HRP injections into the motor and premotor cortices (Kultas-llinsky et al., 1981; Kultas-llinsky et al., unpublished). The large cells contained retrogradely transported HRP, while the small cells were consistently unlabeled. The percentage of labeled cells varied from 50 to $99 \%$ of the total population of large cells, depending on the location of the cortical injection and thalamic sampling sites. These variations were consistent with the topographical organization of thalamocort-

Figure 26. A serial synapse formed by an SR bouton at right, a GAD-containing dendrite $(d)$, and a distal branch of a GAD-negative dendrite ( $D$ ). Another SR bouton at left forms a synapse (solid arrow) with the same GAD-negative dendrite. Asymmetric synapses formed by SR boutons are indicated by solid arrows, and the dendrodendritic symmetric synapse is indicated by an open arrow.

Figure 27. A GAD-positive dendrite (asterisk) in close apposition to a soma of a GAD-negative neuron ( $N$ ). The GAD-positive dendrite is contacted by an SR bouton (arrow).

Figure 28. A small GAD-containing profile identified as an axon terminal (asterisk) forms a symmetric synapse (open arrow) with a GAD-positive dendrite (d). An $\mathrm{SR}$ bouton forms an asymmetric synapse with the same GAD-containing dendrite.

Figure 29. A GAD-positive bouton forms a symmetric contact (arrow) with a soma of a GAD-negative neuron $(N)$ in the VL. $B a r=1 \mu \mathrm{m}$. 

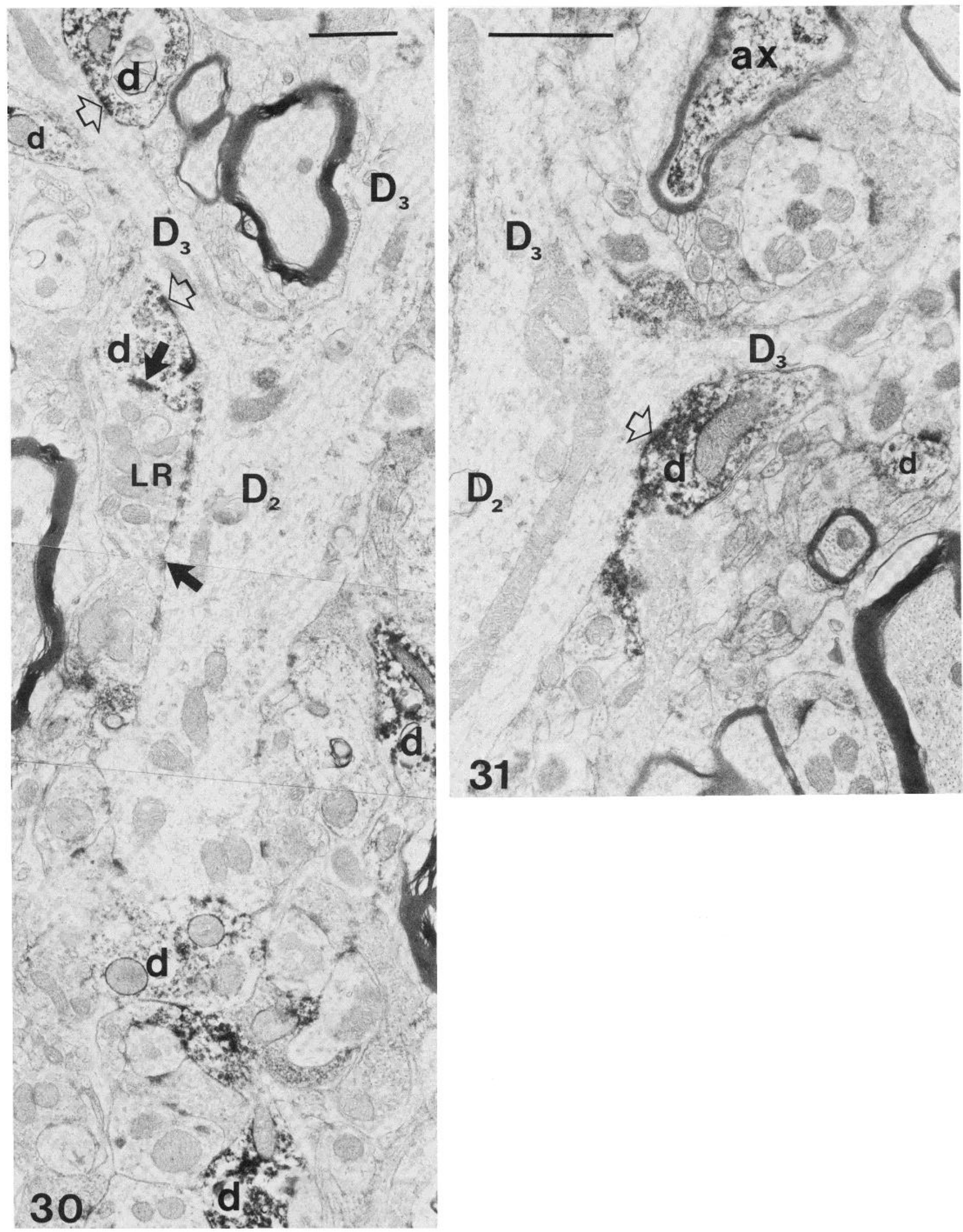

Figures 30 and 31. Low-power electron micrographs illustrating longitudinal sections of secondary and tertiary dendrites of GAD-negative neurons in the VL and their relationship to GAD-containing dendrites. Bars $=1 \mu \mathrm{m}$. 
ical projections from VA, VM, and VL (Bentivoglio et al., 1978; Hendry et al., 1979). The results of these Golgi and HRP studies, together with the present findings, indicate that cells previously suggested to be local circuit neurons are GABAergic, while the large cells that are probably projection neurons are not GABAergic.

These results are consistent with GAD immunocytochemical studies in other thalamic nuclei (Penny et al., 1983; Spreafico et al., 1983; Ohara et al., 1983; Hendrickson et al., 1983; Fitzpatrick et al., 1984). Since a number of unlabeled small cells were observed in the present material, many local circuit neurons might not be GABAergic. The most plausible explanation for not labeling all small cells may be the limited diffusion of the GAD antibodies into the depth of the tissue, resulting in false negatives.

$A$ better understanding of the exact function of these GABAergic neurons in the motor thalamus will come when their axon termination sites are determined. So far, there is very little convincing data to demonstrate the axons of these cells. In the present study, none of the processes arising from the somata of GAD-positive cells were identified as axons. Instead, the processes in continuity with perikarya were invariably found to be postsynaptic to different types of unlabeled axon terminals. Therefore, all of the GAD-positive processes from these small cells were identified as vesicle-containing dendrites which have been well characterized in normal electron microscopic preparations of the VA, VL, and VM (Rinvik and Grofová, 1974a; Kultas-llinsky et al., 1980a).

Golgi studies of VL have shown axons of type II cells that ramify within the limits of the dendritic field of these cells (Kiss and Tömböl, 1972; Rinvik and Grofová, 1974a). However, the examples illustrated in these publications demonstrated only short segments of presumed axons. Moreover, in cases in which these axons were shown to arise from dendrites (Kiss and Tömböl, 1972; Figs. 4 and 6), it was difficult to distinguish them from surrounding dendritic branches of the same cell. It is possible that axons for these cells do exist, but they fail to impregnate properly because they are myelinated. It is also possible that the termination sites and branching of these axons are very limitcd, so that the probability of encountering them in normal electron microscopic preparations is very low. Nevertheless, it should be mentioned that very fine, GAD-positive axons with thin myelin sheaths were frequently observed in the neuropil of all three thalamic nuclei. However, it was not possible to trace them to their sites of origin of termination. In fact, a population of small, GADpositive boutons was present in the VL but was not identified in either VA or VM. Since the Golgi data on axons of small cells were specitic tor $V L$, the existence of fine myelinated GAD-positive axons in VL might lend some support to this notion of local circuit neurons with fine myelinated axons. A final resolution of this problem will only be obtained from a detailed serial section reconstruction of individual local circuit neurons that have received intracellular injections of electron opaque markers following their physiological identification.

Dendrites of GABAergic cells and complex synaptic arrangements. Although it is unclear as to whether or not GABAergic cells in the motor thalamus possess axons, the present findings indicate that the dendrites of these cells play a more significant role in inhibitory synaptic circuitry than their axons. Not only are the GADcontaining dendrites either presynaptic and/or postsynaptic, they are extremely numerous in the neuropil of the three nuclei studied. In addition, these dendrites are contacted directly by terminals of two major afferent systems, the cortico- and cerebellothalamic path- ways, and are involved in feedforward inhibitory circuits with these afferents.

The corticothalamic projections give rise to SR type boutons (Grofová and Rinvik, 1974) which are the most numerous axon terminals in the VA, VM, and VL. They form synapses with both distal dendrites of thalamocortical projection neurons and dendrites of GABAergic cells. The GABAergic dendrites, in turn, are presynaptic to the same distal branches of thalamocortical projection neurons and thus form serial synapses (Fig. 36A). This type of synaptic arrangement explains how a projection neuron dendrite may receive two types of input following the activation of corticothalamic fibers: (1) a direct excitation and (2) feedforward inhibition mediated through GABAergic dendrites. Cortical input may also activate feedforward inhibition of somata of thalamocortical projection neurons by means of serial synapses where a GAD-positive dendrite is postsynaptic to an SR bouton and presynaptic to a soma of a projection neuron.

Cerebellothalamic afferents in VA, VM, and VL give rise to LRtype boutons which commonly participate in another complex synaptic arrangement, the triad (Rinvik and Grofová, 1974b; KultasIlinsky et al., 1980a, 1980b). The present lindings indicale that GABAergic dendrites are also involved in these complexes, where they are postsynaptic to LR boutons and presynaptic to secondary and tertiary dendritic branches of thalamocortical projection neurons. These latter dendrites also receive a direct contact from the same LR bouton (Fig. 36, $B$ and $D$ ). This circuitry helps to explain how an activation of cerebellar fibers will cause a simultaneous excitation of both types of dendrites followed by an inhibition of the thalamocortical projection neuron dendrites.

These two examples suggest that the GABAergic inhibition provided by dendrites of local circuit neurons in the motor thalamus can modulate the activity that arrives via both cortical and cerebellar afferents. This synaptic circuitry may be related to the typical sequences of excitatory and inhibitory postsynaptic potentials that have been recorded from thalamic relay cells after stimulation of both cortico- and ccrcbcllothalamic pathways (Uno ct al., 1970; Frigyesi and Schwartz, 1972; Bava et al., 1979). The GABAergic dendrodendritic synapses that occur on distal dendrites of projection neurons may serve another function as "local semiautonomous computation devices" that integrate the regional input activity on individual dendritic branches (Graubard and Calvin, 1979).

Comparison of $V L$ with VA and VM. The number of LR boutons and associated triads in the regions of the VA and VM appears to be sparse as compared to $\mathrm{VL}$, which receives the major cerebellar input. In the former two nuclei, the LR boutons are almost always associated with GABAergic dendrites in glomeruli or in isolated triads in the neuropil. In contrast, the numerous LR boutons in VL are found either singly along the dendrites of thalamocortical projection neurons, in isolated triads, or in numerous glomeruli. These observations are consistent with quantitative and qualitative descriptions of the synaptic organization of neuropil in VA, VM, and VL (Kultasllinsky et al., 1980a, 1980b; Kultas-llinsky and llinsky, 1983) and the distribution of cerebellar afferents (llinsky and Kultas-llinsky, 1984). This latter study showed that the cerebellar dentate nucleus sends a massive projection to $\mathrm{VL}$, while the remaining deep cerebellar nuclei have only a minor projection to regions of the VA and VM studied here (see "Discussion" in llinsky and Kultas-llinsky, 1984).

The GABAergic presynaptic dendrites associated with cerebellar

Figure 30. Montage of a longitudinally sectioned branching site of a secondary dendrite $\left(D_{2}\right)$ into two tertiary dendrites $\left(D_{3}\right)$ of a GAD-negative cell associated with four GAD-positive dendrites (d). Two of these GAD-containing dendrites form symmetric synapses (open arrows) with the dendrites. One of these GADcontaining dendrites participates in a synaptic triad with a GAD-negative LR type bouton which makes asymmetric synapses (black arrows) with it and a proximal segment of the secondary dendrite $\left(D_{2}\right)$. Part of a glomerulus oriented in a plane perpendicular to $D_{2}$ is seen in the lower part of the micrograph, where several GAD-positive profiles are found.

Figure 31. A GAD-containing dendrite (d) forms a symmetric synapse (open arrow) at the bifurcation site of the GAD-negative dendrite $\left(D_{2}\right)$. To the right of this dendrite is a small GAD-containing profile $(d)$ that receives a synapse from a small SR type bouton (unlabeled). A GAD-containing large myelinated axon is seen in the upper part of the micrograph. $D_{3}$-tertiary dendrites that arise from $D_{2}$. 

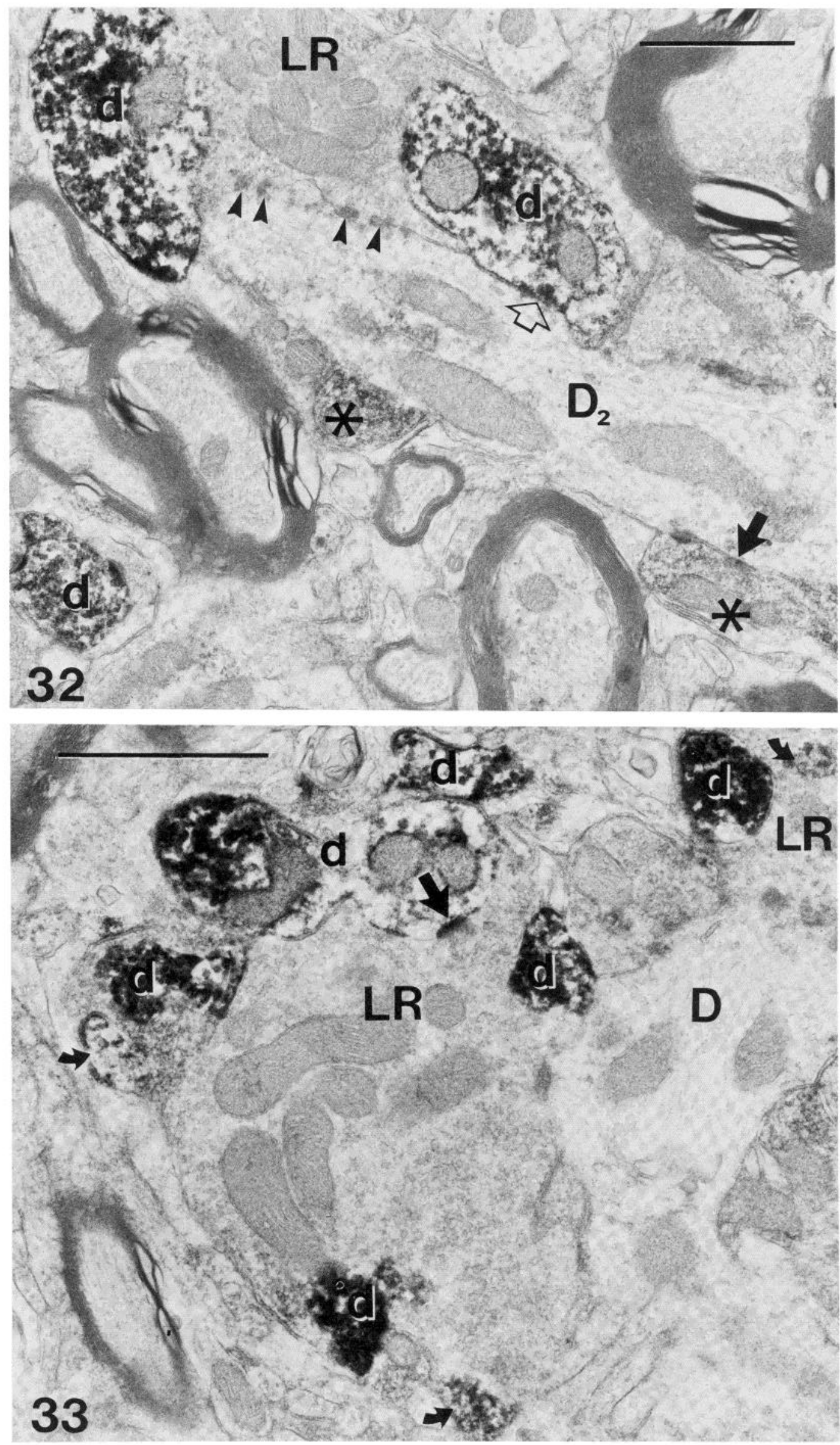

Figures 32 and 33. Enlargements of LR-type boutons associated with synaptic glomeruli in VL. Bars $=1 \mu \mathrm{m}$

Figure 32. An LR bouton is attached with puncta adherentes (small arrowheads) to a secondary dendrite $\left(D_{2}\right)$ of a GAD-negative cell and is apposed to 

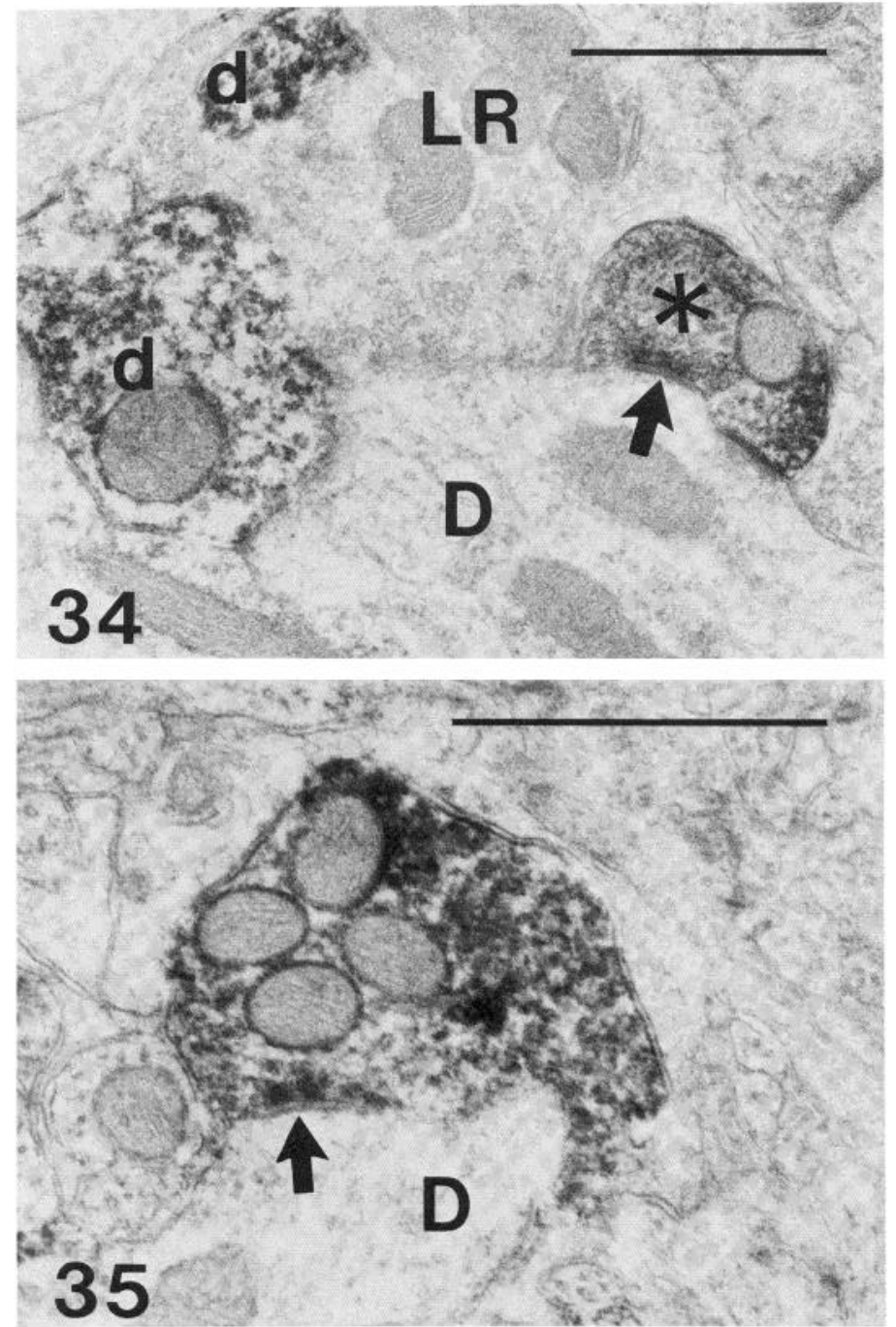

Figures 34 and 35. Examples of GAD-containing boutons in VL. Bars = $1 \mu \mathrm{m}$.

Figure 34. A small GAD-containing terminal (asterisk) forms a symmetric contact (arrow) with a GAD-negative dendrite $(D)$. This bouton is similar to two GAD-containing terminals demonstrated in Figure 32. Adjacent to this GAD-positive bouton are an LR bouton and two GAD-containing dendrites (d).

Figure 35. A medium size GAD-positive bouton that contains several mitochondria and forms a symmetric synapse (arrow) with a GAD-negative dendrite $(D)$. This terminal is similar to the one that contacts a soma in Figure 29.

terminals in VL are most numerous in the glomeruli, where at least two or more GABAergic dendrites are always postsynaptic to the same LR bouton. In addition, these GABAergic dendrites form dendrodendritic synapses at influential positions on the dendrites of thalamocortical projection neurons such as bifurcation sites and bases of primary dendrites. Since these sites are occupied by GABAergic basal ganglia input in VA and VM, the overall influence of GABAergic local circuit neurons on the thalamocortical cells in VL is more significant than in VA and VM. Moreover, the finding that the GABAergic cells in VL receive the cortical input (SR boutons) directly on their somata and proximal dendrites is significant, be cause GABAergic cells in VA and VM do not. Thus, the GABAergic local circuit neurons in VL are probably driven more by corticothalamic fibers than similar cells in VA and VM.

GABAergic terminals. Although most GAD-positive, vesicle-containing profiles in VL were dendrites, two types of GABAergic terminals were also present. The larger type of terminal did not exhibit any preferential location and was encountered infrequently either on somata or on different order dendrites of thalamocortical projection neurons. In contrast, the small GAD-positive boutons were associated with the LR boutons and glomeruli and shared the same postsynaptic dendrites. Unfortunately, the present data do not determine the exact source of these axon terminals in the VL. In fact, both types of boutons may be derived from the thalamic reticular nucleus because the axon terminals from this nucleus display considerable variation in size and morphology in the rat lateral geniculate nucleus (Ohara et al., 1980; Montero and Scott, 1981). If only limited interspecies and internuclear differences exist between the rat and cat thalamus, then it is possible that both types of GAD-containing boutons in the VL are of thalamic reticular origin.

In contrast to VL, only one type of GABAergic terminal was observed in VA and anterolateral VM, and this type displayed the features of basal ganglia afferents. It is possible that GABAergic terminals derived from the thalamic reticular nucleus and local circuit neurons may exist in these nuclei (see discussion above), but their ultrastructural features and synaptic sites would have to be similar to those of basal ganglia terminals. A possibility that we favor is that thalamic regions which receive overlapping nigral and pallidal projections have no appreciable numbers of GABAergic axons from other sources. A corollary to this notion would state that a thalamic region which receives basal ganglia afferents from only one source may have GABAergic terminals derived from other non-basal ganglia sites. Data from the medial VM, which receives only one input from the basal ganglia, support the corollary and lend evidence to this hypothesis. For example, nigrothalamic fibers form synapses with the bases of primary dendrites but not with the somata of thalamocortical neurons (Kultas-llinsky et al., 1983). Since GAD-containing boutons that display a morphology different from that of basal ganglia boutons were observed infrequently to form synapses directly with somata of thalamocortical neurons, these GABAergic axosomatic terminals in medial VM are probably derived from sources other than the substantia nigra.

Finally, synapses between two GABAergic structures are considered to be an anatomical substrate for disinhibition (Roberts, 1976). Such synapses were expected in the VA and VM because a modest number of basal ganglia afferents in these nuclei contact vesiclecontaining dendrites (Kultas-llinsky et al., 1983). Although the present study has demonstrated numerous examples of close appositions of GAD-positive boutons to GAD-positive dendrites, the dense accumulations of the immunoreaction product in these profiles obscured the unequivocal demonstration of synaptic contacts between these structures in the VA and VM. However, some convincing examples of contacts between GAD-containing axons and dendrites were found in the VL. Since the origin of GABAergic axons in the $\mathrm{VL}$ is unknown, the functional implication of this synaptic relationship remains to be understood. Nevertheless, these findings indicate that the anatomical substrate for disinhibition mediated by GABAergic dendrites exists in the motor thalamus.

Conclusions. Although additional studies are needed to clarify a number of questions that arise from the present investigation, it is clear that the cat motor thalamus contains inhibitory, GABAergic circuits that are complex and differ according to the individual

two GAD-containing dendrites $(d)$. One of these dendrites forms a synaptic contact (open arrow) with the GAD negative dendrite $\left(D_{2}\right)$. Two small GADpositive profiles (asterisks) identified as axon terminals are also apposed to this GAD-negative dendrite $\left(D_{2}\right)$, and one of them forms a symmetric synapse (arrow).

Figure 33. Another glomerulus with both a centrally located LR bouton and a second LR bouton in the upper right. Six GAD-containing dendrites ( $d$ and curved arrows) are associated with the central LR bouton, and one dendrite displays a synaptic contact in the section plane (arrow). D: dendrite of a GADnegative neuron. 
A
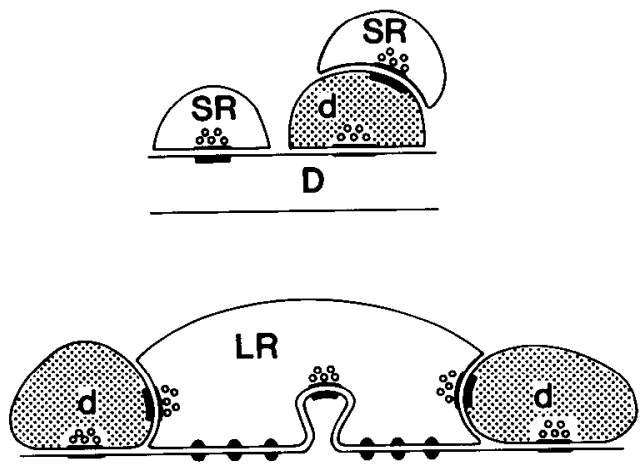

B

D

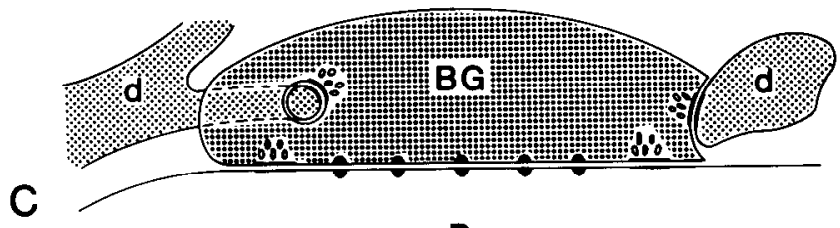

D

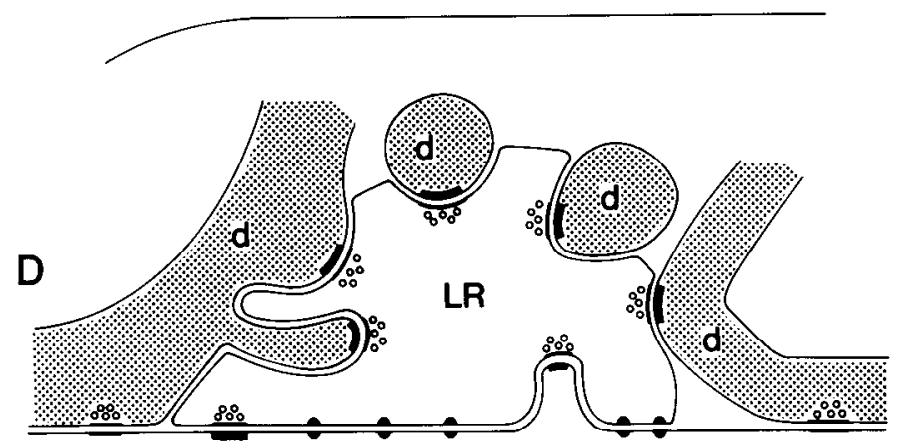

D

an increased number of local circuit inhibitory neurons in the brain (see "Discussion" by Rakic, 1976). This trend is evident for the motor nuclei of the thalamus, which in the cat possess a great degree of complexity compared to those in the rat, where neurons lack vesiclecontaining dendrites (Kultas-llinsky, unpublished observations). This lrend is further borne out in the primate motor thalamus, which is similar to that of the cat, but has in addition complex synaptic arrangements with involvement of vesicle-containing dendrites of a kind not found in the cat (Harding and Powell, 1977). Therefore, generalizations, or direct extrapolations of data on inhibitory mechanisms in the cat thalamus to the primate, and especially the human brain, should be approached with extreme caution. Nevertheless, it is apparent that under pathological conditions, such as Huntington's chorea or Parkinsonism, the net changes in the output activity of thalamocortical neurons would result not only from the primary impairment or elimination of inhibitory, basal ganglia inputs to these cells but would also reflect the shifted balances in local inhibitory circuits caused by such impairments.

\section{References}

Bava, A., F. Cicirata, S. Licciardello, G. L. Volsi, and M. R. Panto (1979) Fastigial nuclei projections on the ventralis lateralis $(V L)$ thalamic nucleus neurons. Brain Res. 168: 169-175.

Bentivoglio, M., P. Rossini, G. Macchi, and E. Tempesta (1978) Le proiezioni thalamocorticali del nucleo ventrale anteriore. Studio mediante il transporto assonale retrogrado di HRP nel gatto. Arch. Ital. Anat. Embriol. 83: 111127

Deniau, J. M., D. Lackner, and J. Feger (1978) Effect of substantia nigra stimulation on identified neurons in the VL-VA thalamic complex: comparison between intact and chronically decorticated cats. Brain Res. 145: 27-35.

Di Chiara, G., M. L. Porceddu, M. Morelli, M. L. Mulas, and G. L. Gessa (1979) Evidence for a GABAergic projection from the substantia nigra to the ventromedial thalamus and to the superior colliculus of the rat. Brain Res. 176: 273-284.

Fahn, A. (1976) Regional distribution studies of GABA and other putative neurotransmitters and their enzymes. In GABA in Nervous System Function, E. Roberts, T. N. Chase, and D. B. Tower, eds., pp. 169-186, Raven Press, New York.

Fitzpatrick, D., G. R. Penny, and D. E. Schmechel (1984) Glutamic acid decarboxylase-immunoreactive neurons and terminals in the lateral geniculate nucleus of the cat. J. Neurosci. 4: 1809-1829.

Frigyesi, T. L., and R. Schwartz (1972) Cortical control of thalamic sensorimotor relay activity in the cat and squirrel monkey. In Corticothalamic Projections and Sensorimotor Activities, T. L. Frigyesi, E. Rinvik, and M. D. Yahr, eds., pp. 161-195, Raven Press, New York.

Figure 36. A schematic diagram of the major types of synaptic relationships that involve afferent terminals and GABAergic dendrites in the motor nuclei of the thalamus. $A$, Serial synapse formed by an SR bouton (cortical afferent), a GAD-positive (shaded) dendrite (d) of a local circuit neuron, and a GAD-negative dendrite $(D)$ of a thalamocortical projection neuron. The additional SR bouton on the left is not a part of the serial synapse but is usually found in the vicinity of serial arrays, where it forms a synaptic contact with the same projection neuron dendrite. Serial synapses are characteristic for all three motor nuclei of the thalamus. $B$, A triad formed by an LR-type bouton (cerebellar origin), two GAD-containing (shaded) dendrites of local circuit neurons $(d)$, and a GAD-negative dendrite of a thalamocortical projection neuron $(D)$. Such synaptic relationship is found in VA and VM and, to a lesser extent, in VL. C, A GAD-containing (shaded) bouton of basal ganglia origin $(B G)$ is shown to form synapses with a GAD-negative dendrite of a thalamocortical projection neuron $(D)$ and also with two GAD-containing dendrites of local circuit neurons (d). Such synaptic relationships are found in VA and VM but not in VL, which lacks basal ganglia afferents. $D, A$ centrally located LR-type bouton forms multiple synaptic contacts with numerous GAD-containing dendrites of local circuit neurons $(d)$ and with a GAD-negative dendrite of a thalamocortical projection neuron $(D)$. Such glomeruli are numerous in VL but are not present in the VA and VM. For all drawings, asymmetric synapses are indicated by vesicle accumulations and a thick postsynaptic density, while symmetric synapses lack the density.

nucleus studied. These circuits are formed by incoming afferents as well as intrinsic GABAergic cells, and both contribute to the shaping of the output activity of thalamocortical projection neurons. It is interesting to note that there is probably an evolutionary trend toward

Graubard, K., and W. H. Calvin (1979) Presynaptic dendrites: implications of spikeless synaptic transmission and dendritic geometry. In The Neurosciences: Fourth Study Program, F. D. Schmitt and F. G. Worden, eds., pp. 317-331, MIT Press, Cambridge, MA.

Grofová, I., and E. Rinvik (1974) Cortical and pallidal projections to the nucleus ventralis lateralis thalami. Electron microscopic studies in the cat. Anat. Embryol. (Berl.) 146: 113-132.

Harding, B. N., and T. P. S. Powell (1977) An electron microscopic study of the centre-median and ventrolateral nuclei of the thalamus in the monkey. Philos. Trans. R. Soc. Lond. (Biol.) 279: 357-412.

Hendrickson, A. E., M. P. Ogren, J. E. Vaughn, R. P. Barber, and J. -Y. Wu (1983) Light and electron microscopic immunocytochemical localization of glutamic acid decarboxylase in monkey geniculate complex: Evidence for GABAergic neurons and synapses. J. Neurosci. 3: 1245-1262.

Hendry, S. H. C., E. G. Jones, and J. Graham (1979) Thalamic relay nuclei for cerebellar and certain related fiber systems in the cat. J. Comp. Neurol. 185: 679-714.

Houser, C. R., J. E. Vaughn, R. P. Barber, and E. Roberts (1980) GABA neurons are the major cell type of the nucleus reticularis thalami. Brain Res. 200: 341-354.

Hsu, S. M., L. Raine, and H. Fanger (1981) Use of Avidin-Biotin-Peroxidase Complex $(\mathrm{ABC})$ in immunoperoxidase techniques: a comparison between $A B C$ and unlabeled antibody (PAP) procedures. $J$ Histochem. Cytochem. 29: $577-580$.

llinsky, I. A., K. Kultas-llinsky, and K. R. Smith (1982) Organization of the basal ganglia inputs to the thalamus. A light and electron microscopic study in the cat. Appl. Neurophysiol. 45: 230-237.

llinsky, I. A., and K. Kultas-llinsky (1984) An autoradiographic study of 
topographical relationships between pallidal and cerebellar projections to the cat thalamus. Exp. Brain Res. 54: 95-105.

Jones, E. G. (1975) Some aspects of the organization of the thalamic reticular complex. J. Comp. Neurol. 162: 285-308.

Jones, E. G., and T. P. S. Powell (1969) Electron microscopy of synaptic glomeruli in the thalamic relay nuclei in the cat. Proc. R. Soc. Lond. (Biol.) 172: 153-171.

Kilpatrick, I. C., M. S. Starr, A. Fletcher, T. A. James, and N. K. McLeod (1980) Evidence for a GABAergic nigrothalamic pathway in the rat. I Behavioral and biochemical studies. Exp. Brain Res. 40: 45-54.

Kiss, A., and T. Tömböl (1972) Golgi analysis and degeneration studies of the nucleus ventralis lateralis and ventralis medialis in the cat thalamus. Brain Res. 47: 303-315

Kultas-llinsky, K., I. A. linsky, L. C. Massopust, P. A. Young, and K. R. Smith (1978) Nigrothalamic pathway in the cat derronstrated by auloradiography and electron microscopy. Exp. Brain Res. 33: 481-492.

Kultas-llinsky, K., I. A. linsky, P. A. Young, and K. R. Smith (1980a) Ultrastructure of degenerating cerebellothalamic terminals in the ventral medial nucleus of the cat. Exp. Brain Res. 38: 125-135.

Kultas-llinsky, K., S. Warton, D. L. Tolbert, and I. A. llinsky (1980b) Quantitative and qualitative characteristics of dentate and fastigial afferents identified by electron microscopic autoradiography in the cat thalamus. Brain Res. 201: $220-226$.

Kultas-llinsky, K. S. Warton, and I. A. llinsky (1981) Light and electron microscopical features of the types of neurons in the cat ventral medial thalamic nucleus as revealed with HRP injections in the motor cortex. Soc. Neurosci. Abstr. 7: 416.

Kultas-llinsky, K., and I. A. llinsky (1983) Subdivisions of motor related thalamic nuclei in the cat based on their connectivity and ultrastructure. Soc. Neurosci. Abstr. 9: 1230.

Kultas-llinsky, K., I. llinsky, S. Warton, and K. R. Smith (1983) Fine structure of nigral and pallidal afferents in the thalamus: An EM autoradiography study in the cat. J. Comp. Neurol. 216: 390-405.

McLeod, N. K., T. A. James, I. C. Kilpatrick, and M. S. Starr (1980) Evidence for GABAergic nigrothalamic pathway in the rat. II. Electrophysiological studies. Exp. Brain Res. 40: 55-61

Montero, V. M. and G. L. Scott (1981) Synaptic terminals in the dorsal lateral geniculate nucleus from neurons of the thalamic reticular nucleus: A light and electron microscope autoradiographic study. Neuroscience 6: 25612577

Oertel, W. H., D. E. Schmechel, M. L. Tappaz, and I. J. Kopin (1981a) Production of a specific antiserum to rat brain glutamic acid decarboxylase by injection of an antigen-antibody complex. Neuroscience 6: 2689-2700.

Oertel, W. H., D. E. Schmechel, E. Mugnaini, M. L. Tappaz, and I. J. Kopin (1981b) Immunocytochemical localization of glutamate decarboxylase in rat cerebellum with a new antiserum. Neuroscience 6: 2715-2735.

Oertel, W. H., A. M. Graybiel, E. Mugnaini, R. P. Elde, D. E. Schmechel, and I. S. Kopin (1983) Coexistence of glutamic acid decarboxylase- and somatostatin-like immunoreactivity in neurons of the feline nucleus reticularis thalami. J. Neurosci. 3: 1322-1332.

Oertel, W. H., C. Nitsch, and E. Mugnaini (1984) Immunocytochemical demonstration of the GABAergic neurons in rat globus pallidus and nucleus entopeduncularis and their GABA-ergic innervation. Adv. Neurol. 40: 9198.

Ohara, P. T., A. J. Sefton, and A. R. Lieberman (1980) Mode of termination of afferents from the thalamic reticular nucleus in the dorsal lateral geniculate nucleus of the rat. Brain Res. 197: 503-506.
Ohara, P. T., A. R. Lieberman, S. P. Hunt, and J. -Y. Wu (1983) Neural elements containing glutamic acid decarboxylase (GAD) in the dorsa lateral geniculate nucleus of the rat: Immunohistochemical studies by light and electron microscopy. Neuroscience 8: 189-211.

Ordronneau, P., P. B. M. Lindstrom, and P. Petrusz (1981) Four unlabeled antibody bridge techniques: A Comparison. J. Histochem. Cytochem. 29 1397-1404

Penney, J. B., Jr., and A. B. Young (1981) GABA as the pallidothalamic neurotransmitter: Implications for basal ganglia function. Brain Res. 207: 195-199.

Penny, G. R., D. Fitzpatrick, D. E. Schmechel, and I. T. Diamond (1983) Glutamic acid decarboxylase-immunoreactive neurons and horseradish peroxidase-labeled projection neurons in the ventral posterior nucleus of the cat and Galago Senegalensis. J. Neurosci. 3: 1868-1887.

Purpura, D. P. (1972) Intracellular studies of synaptic organization in the mammalian brain. In Structure and Function of Synapses, G. D. Pappas and D. P. Purpura, eds., pp. 257-302, Raven Press, New York.

Rakic, P. (1976) Local Circuit Neurons, MIT Press, Cambridge, MA

Ribak, C. E., J. E. Vaughn, and K. Saito (1978) Immunocytochemical localization of glutamic acid decarboxylase in neuronal somata following colchicine inhibition of axonal transport. Brain Res. 140: 315-332.

Ribak, C. E., J. E. Vaughn, and R. P. Barber (1981) Immunocytochemical localization of GABAergic neurones at the electron microscopical level. Histochem. J. 13: 555-582.

Rinvik, E., and I. Grofová (1974a) Light and electron microscopical studies of the normal nuclei ventralis lateralis and ventralis anterior thalami in the cat. Anat. Embryol. (Berl.) 146: 57-93.

Rinvik, E., and I. Grofová (1974b) Cerebeliar projections to the nuclei ventralis lateralis and ventralis anterior thalami. Experimental electron microscopica and light microscopical studies in the cat. Anat. Embryol. (Berl.) 146: 95111

Roberts, E. (1976) Disinhibition as an organizing principle in the nervous system: The role of the GABA system. Application to neurologic and psychiatric disorders. In GABA in Nervous System Function, E. Roberts, T. N. Chase, and D. B. Tower, eds., pp. 515-539, Raven Press, New York

Scheibel, M. E., and A. B. Scheibel (1966) The organization of the nucleus reticularis thalami: A Golgi study. Brain Res. 1: 43-62.

Schlag, J., and M. Waszak (1970) Characteristics of unit responses in nucleus reticularis thalami. Brain Res. 21: 286-288.

Spreafico, R., D. E. Schmechel, L. C. Ellis, Jr., and A. Rustioni (1983) Cortical relay neurons and interneurons in the $\mathrm{N}$. ventralis posterolateralis of cats: A horseradish peroxidase, electron-microscopic, Golgi and immunocytochemical study. Neuroscience 9: 491-509.

Starr, M. S., and I. C. Kilpatrick (1981) Distribution of $\gamma$-aminobutyrate in the rat thalamus: specific decreases in thalamic $\gamma$-aminobutyrate following lesion or electrical stimulation of the substantia nigra. Neuroscience 6 . 1095-1104.

Ueki, A. (1983) The mode of nigrothalamic transmission investigated with intracellular recording in the cat. Exp. Brain Res. 49: 116-124.

Uno, M. M. Yoshida, and I. Hirota (1970) The mode of cerebellothalamic relay transmission investigated with intracellular recording from the cells of the ventrolateral nucleus of cat's thalamus. Exp. Brain Res. 10: 121-139.

Uno, M., N. Ozawa, and M. Yoshida (1978) The mode of pallido-thalamic transmission investigated with intracellular recording from cat thalamus. Brain Res. 33: 493-507. 